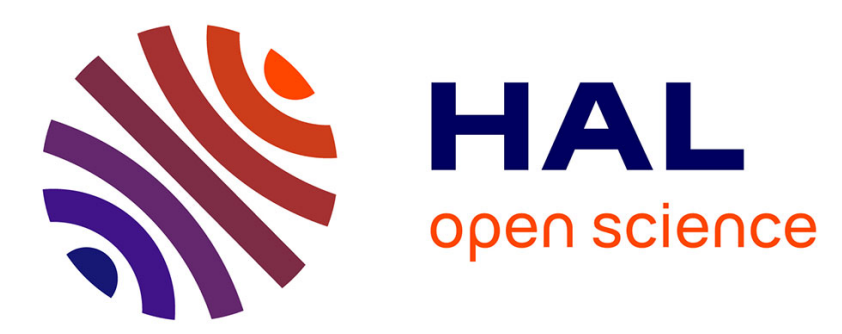

\title{
Elliptic eigenvalue problems with large drift and applications to nonlinear propagation phenomena
}

Henri Berestycki, Francois Hamel, Nikolai Nadirashvili

\section{To cite this version:}

Henri Berestycki, Francois Hamel, Nikolai Nadirashvili. Elliptic eigenvalue problems with large drift and applications to nonlinear propagation phenomena. Communications in Mathematical Physics, 2005, 253, pp.451-480. hal-00003740

\section{HAL Id: hal-00003740 \\ https://hal.science/hal-00003740}

Submitted on 2 Jan 2005

HAL is a multi-disciplinary open access archive for the deposit and dissemination of scientific research documents, whether they are published or not. The documents may come from teaching and research institutions in France or abroad, or from public or private research centers.
L'archive ouverte pluridisciplinaire HAL, est destinée au dépôt et à la diffusion de documents scientifiques de niveau recherche, publiés ou non, émanant des établissements d'enseignement et de recherche français ou étrangers, des laboratoires publics ou privés. 


\title{
Elliptic eigenvalue problems with large drift and applications to nonlinear propagation phenomena
}

\author{
Henri Berestycki ${ }^{\mathrm{a}}$, François Hamel ${ }^{\mathrm{b}}$ and Nikolai Nadirashvili ${ }^{\mathrm{c} *}$ \\ a EHESS, CAMS, 54 Boulevard Raspail, F-75006 Paris, France \\ b Université Aix-Marseille III, LATP, Faculté Saint-Jérôme, Case cour A \\ Avenue Escadrille Normandie-Niemen, F-13397 Marseille Cedex 20, France \\ c University of Chicago, Department of Mathematics, \\ 5734 University Avenue, Chicago, IL 60637-1546, USA
}

\begin{abstract}
This paper is concerned with the asymptotic behavior of the principal eigenvalue of some linear elliptic equations in the limit of high first-order coefficients. Roughly speaking, one of the main results says that the principal eigenvalue, with Dirichlet boundary conditions, is bounded as the amplitude of the coefficients of the first-order derivatives goes to infinity if and only if the associated dynamical system has a first integral, and the limiting eigenvalue is then determined through the minimization of the Dirichlet functional over all first integrals. A parabolic version of these results, as well as other results for more general equations, are given. Some of the main consequences concern the influence of high advection or drift on the speed of propagation of pulsating travelling fronts.
\end{abstract}

\section{Introduction}

Nonlinear propagation of fronts in reaction-diffusion equation of the Fisher type often involves a linear eigenvalue problem. In particular, it is of interest to study the effects of various phenomena such as diffusion, reaction, advection on the speed of fronts. Carrying this rests on some singular limits in these eigenvalue problems. This paper is concerned with both aspects. We derive here some limiting behaviour of eigenvalue problems and we also establish results for nonlinear propagation phenomena.

To illustrate the type of results for the first aspect, let us consider the simple case of

\footnotetext{
*The third author was partially supported by a NSF grant
} 
Dirichlet eigenvalue problem for the Laplacian with a large divergence free drift. Consider a bounded domain $\Omega \subset \mathbb{R}^{N}$ of class $C^{2}$, with outward unit normal $\nu=\nu(x)$ (for $x \in \partial \Omega$ ). Let $v$ be a $L^{\infty}(\Omega)$ vector field, such that

$$
\operatorname{div} v=0 \text { in } \mathcal{D}^{\prime}(\Omega)
$$

and, for $A \in \mathbb{R}$, let $\lambda_{A}$ be the principal eigenvalue and $\varphi_{A}$ be the principal eigenfunction (unique up to multiplication) of

$$
\left\{\begin{aligned}
-\Delta \varphi_{A}+A v \cdot \nabla \varphi_{A} & =\lambda_{A} \varphi_{A} & & \text { in } \Omega \\
\varphi_{A} & =0 & & \text { on } \partial \Omega .
\end{aligned}\right.
$$

We recall that $\varphi_{A} \in W^{2, p}(\Omega)$ for all $1 \leq p<+\infty$ (hence $\varphi_{A} \in C^{1, \alpha}(\bar{\Omega})$ for all $0 \leq \alpha<1$ ), and that one can assume that $\varphi_{A}>0$ in $\Omega$.

Our purpose here is to analyze the limiting behavior of the first eigenvalue $\lambda_{A}$ in the limit $A \rightarrow+\infty$ (note that through a change of $v$ into $-v$, the limit $A \rightarrow-\infty$ is treated similarly). As was already mentioned, the goal is to understand the influence of high drift coefficients. In doing this, one has to understand the role of advection and that of diffusion.

The limiting behavior of the solutions of problem (0.1) as $A \rightarrow+\infty$ turns out to be strongly related to the existence of first integrals of $v$ in $H_{0}^{1}(\Omega)$. We now define this notion :

Definition 0.1 A function $w$ is said to be a first integral of the vector field $v$ if $w \in$ $H^{1}(\Omega), w \neq 0$ and $v \cdot \nabla w=0$ almost everywhere in $\Omega$.

Definition 0.2 We note $\mathcal{I}_{0}$ the set of all first integrals of $v$ which belong to $H_{0}^{1}(\Omega)$.

Notice that if both $v$ and a first integral $w$ are smooth enough, then $w$ is constant along the trajectories of the dynamical system $\dot{X}=v(X)$ in $\bar{\Omega}$.

Theorem 0.3 The first eigenvalues $\left(\lambda_{A}\right)$ of (0.1) are bounded as $A \rightarrow+\infty$ if and only if $v$ has a first integral in $H_{0}^{1}(\Omega)$. Furthermore,

1) If $v$ does not have any first integral in $H_{0}^{1}(\Omega)$, then $\lambda_{A} \rightarrow+\infty$ as $A \rightarrow+\infty$.

2) If $v$ has a first integral in $H_{0}^{1}(\Omega)$, then

$$
\lambda_{A} \rightarrow \min _{w \in \mathcal{I}_{0}} \frac{\int_{\Omega}|\nabla w|^{2}}{\int_{\Omega} w^{2}} \text { as } A \rightarrow+\infty,
$$

and the minimum in the right-hand side of (0.2) is achieved. 
From the proof, we will see that furthermore,

$$
\lambda_{A} \leq \frac{\int_{\Omega}|\nabla w|^{2}}{\int_{\Omega} w^{2}}
$$

for all $A \in \mathbb{R}$ and for all $w \in \mathcal{I}_{0}$.

Theorem 0.3 gives a necessary and sufficient condition for the eigenvalues $\lambda_{A}$ 's stay bounded as $A \rightarrow+\infty$. Formula (0.3) also says that the eigenvalues $\lambda_{A}$ are never larger than the Rayleigh quotient of a first integral, if any. A sufficient condition for the boundedness of $\lambda_{A}$, stronger than the existence of first integrals (requiring additional properties for some first integrals), was given by Devinatz, Ellis and Friedman [13]. A large literature has been devoted to this type of question, which we recall in Section 1. Nevertheless, as far as we know, the necessary and sufficient condition for the boundedness of $\lambda_{A}$, and the limiting behavior of $\lambda_{A}$ given by (0.2), had not been observed until now.

More general eigenvalue problems with large drift, as well as operators with other boundary conditions, are also dealt with in Section 2. For instance, Neumann or periodic boundary conditions are considered.

Applications to the decay of solutions of the heat equation with large drift, under Dirichlet boundary conditions, are given in Section 3.

Lastly, some applications to nonlinear propagation phenomena are given in Section 4. One is especially concerned with propagation of fronts for some reaction-diffusion equations of the type

$$
u_{t}-\operatorname{div}(a \nabla u)+A v \cdot \nabla u=f(u)
$$

in periodic media. Under some assumptions on $f$ and on the other parameters, which are recalled in Section 4, it is known that there exist some pulsating (or periodic) travelling fronts propagating in any given direction in which the domain is unbounded. One especially gives in Section 4 a necessary and sufficient condition for the minimal speed of pulsating fronts be asymptotically at least linear with respect to the amplitude $A$ of the drift as $A \rightarrow+\infty$. This condition involves the first integrals of the vector field $v$. Applications to reaction-diffusion equations with large reaction or small diffusion are also given.

\section{The case of Dirichlet boundary conditions}

This section is mainly devoted to the proof of Theorem 0.3. Before proceeding to the proof, let us examine some particular cases and briefly recall the literature which has been devoted to this type of problem.

Let us first mention some particular cases where the field $v$ has first integrals. For instance, if $v$, say, vanishes on an open subset $\omega \subset \subset \Omega$, then $v$ has first integrals : namely, any nonzero $w \in H_{0}^{1}(\omega)$, extended by 0 in $\Omega \backslash \omega$, is a first integral of $v$; furthermore, 
under the same conditions, if follows that $\lambda_{A}$ is not larger than the first eigenvalue of the Laplace operator in $\omega$ with Dirichlet boundary conditions (the latter agrees with the monotonicity of the first eigenvalue of an elliptic operator with respect to the domain, see $[8])$.

Notice that if $v$ and a first integral $w$ are respectively $C^{1}$ and continuous in a neighbourhood of some point $x_{0} \in \partial \Omega$, and if $\nabla w\left(x_{0}\right) \neq 0$, then $v\left(x_{0}\right) \cdot \nu\left(x_{0}\right)=0$. Nevertheless, as a consequence of what was just mentioned, this condition $v \cdot \nu=0$ on $\partial \Omega$ is not necessary in general for the existence of a first integral $w$. On the other hand, in dimension $N=2$, if a $C^{1}(\bar{\Omega})$ vector field $v$ satisfies $v \cdot \nu=0$ on $\partial \Omega$, together with $\operatorname{div} v=0$ in $\Omega$, then it is easy to see that $v$ has first integrals.

In the case where $v$ does not have any first integral, the asymptotic rate of growth of $\lambda_{A}$ to $+\infty$ as $A \rightarrow+\infty$ is not known in general. However, for general continuous $v$ without the divergence-free assumption, the limit of $\lambda_{A} / A^{2}$ is known explicitely. It is given by the following formula due to Wentzell [37] :

$$
\frac{\lambda_{A}}{A^{2}} \underset{A \rightarrow+\infty}{\longrightarrow} \frac{1}{4} \lim _{T \rightarrow+\infty}\left[\frac{1}{T} \inf \left(\int_{0}^{T}|\dot{X}(t)+v(X(t))|^{2} d t\right)\right],
$$

where the infimum is taken over all $C^{1}$ functions $X:[0, T] \rightarrow \bar{\Omega}$. It follows in particular that if the dynamical system $\dot{X}=-v(X)$ has a trajectory which stays in $\bar{\Omega}$ for all $t \geq 0$, then $\lambda_{A}=o\left(A^{2}\right)$ as $A \rightarrow+\infty$. In particular, $\lambda_{A}=o\left(A^{2}\right)$ if $N=2, v \in C(\bar{\Omega})$ and $v \cdot \nu=0$ on at least one connected component of $\partial \Omega$. This also holds in any dimension if $v$ is continuous and has at least one nonzero first integral $w$ in $C(\bar{\Omega}) \cap C^{1}(\Omega)$ that vanishes on $\partial \Omega$. The last results also hold in the case div $v \neq 0$. In the case of existence of first integrals in $H_{0}^{1}(\Omega)$, together with the divergence-free assumption but without the continuity assumption for $v$, Theorem 0.3 is much more precise. It says further that the $\lambda_{A}$ 's are bounded and their limiting behavior is given.

Formula (1.1) implies that, as soon as $v$ is continuous but may or may not be divergence-free, one has $\lambda_{A}=O\left(A^{2}\right)$ as $A \rightarrow+\infty .^{1}$ In some special cases, one can say that $\lambda_{A}$ behaves likes $A^{2}$ as $A \rightarrow+\infty$. For instance, if $v$ is a gradient field which does not vanish in $\bar{\Omega}$, namely if there exists $U \in C^{2}(\bar{\Omega})$ such that $v=\nabla U$ and $v \neq 0$ in $\bar{\Omega}$, and if $\varphi_{A}$ is an eigenfunction of (0.1) for the first eigenvalue $\lambda_{A}$, then the function $\psi_{A}(x)=e^{-\frac{A}{2} U(x)} \varphi_{A}(x)$ is an eigenfunction of the following self-adjoint problem

$$
\left\{\begin{aligned}
-\Delta \psi_{A}+\left(\frac{A^{2}}{4}|v|^{2}-\frac{A}{2} \operatorname{div} v\right) \psi_{A} & =\lambda_{A} \psi_{A} & & \text { in } \Omega \\
\psi_{A} & =0 & & \text { on } \partial \Omega .
\end{aligned}\right.
$$

\footnotetext{
${ }^{1}$ Actually, it is simple to see, using the change of variables $x^{\prime}=A\left(x-x_{0}\right)$, that $\lambda_{A}=O\left(A^{2}\right)$ as soon as $v$ is continuous at a point $x_{0} \in \Omega$.
} 
Hence,

$$
\lambda_{A}=\min _{\phi \in H_{0}^{1}(\Omega) \backslash\{0\}} \frac{\int_{\Omega}|\nabla \phi|^{2}+\left(\frac{A^{2}}{4}|v|^{2}-\frac{A}{2} \operatorname{div} v\right) \phi^{2}}{\int_{\Omega} \phi^{2}} \sim \frac{A^{2}}{4} \min _{\bar{\Omega}}|v|^{2}
$$

as $A \rightarrow+\infty$, since $v \neq 0$ in $\bar{\Omega}$. More generally speaking, $\lambda_{A} \geq c A^{2}$ for some positive constant $c$ if there exists a $C^{1}(\bar{\Omega})$ function $\phi$ such that $v \cdot \nabla \phi>0$ in $\bar{\Omega}$ (see [13]).

On the other hand, $\lambda_{A}=O(A)$ as soon as $v$ is continuous and vanishes at a point $x_{0} \in \Omega$. More generally speaking, any upper bound like $A^{\alpha}$ with any positive $\alpha$ can be obtained for the $\lambda_{A}$ 's (see [13]). Let us lastly mention that other results on the asymptotics of the first eigenvalues of some elliptic problems set in the whole space $\mathbb{R}^{N}$ are given in [17].

Remark 1.1 (Case of a field which is not divergence free) As already said, $\lambda_{A}$ behaves at most like $A^{2}$ as $A \rightarrow+\infty$, as soon as $v$ is continuous at a point in $\Omega$, but with or without the divergence-free assumption. Furthermore, $\lambda_{A}$ is always nonnegative, from the maximum principle. However, Theorem 0.3 does not hold if $v$ is not divergence-free. For instance, if $v$ is continuous in $\bar{\Omega}$ and $v \cdot \nu>0$ on $\partial \Omega$, then $\lambda_{A}=O\left(A e^{-\alpha A}\right)$ as $A \rightarrow+\infty$, for some constant $\alpha>0$ (see Friedman [20]). In particular, in a ball $B$ with center 0 , for the velocity field $v=x$, one has $\lambda_{A} \rightarrow 0$ and $v$ does not have any first integral in $H_{0}^{1}(\Omega)$. Notice that Theorem 0.3 also says that $\lambda_{A}$ converges either to $+\infty$ or to a positive constant if $v$ is divergence-free.

We now turn to the

Proof of Theorem 0.3. We divide it into the next two lemmas from which Theorem 0.3 obviously follows :

Lemma 1.2 Let $\left(A_{n}\right)_{n \in \mathbb{N}}$ be a sequence such that $A_{n} \rightarrow+\infty$ and $\left(\lambda_{A_{n}}\right)_{n \in \mathbb{N}}$ is bounded. Assume furthermore that the principal eigenfunctions $\varphi_{A_{n}}$ are normalized with $\left\|\varphi_{A_{n}}\right\|_{L^{2}(\Omega)}=1$ for all $n$. Then there exists a subsequence $n^{\prime} \rightarrow+\infty$ and a function $w \in \mathcal{I}_{0}$ such that $\varphi_{A_{n^{\prime}}} \rightarrow w$ strongly in $L^{2}$ and weakly in $H^{1}$, and

$$
\liminf _{n^{\prime} \rightarrow+\infty} \lambda_{A_{n^{\prime}}} \geq \int_{\Omega}|\nabla w|^{2} / \int_{\Omega} w^{2}=\int_{\Omega}|\nabla w|^{2} .
$$

In particular, $\mathcal{I}_{0} \neq \emptyset$.

Lemma 1.3 Assume that $v$ has at least one first integral in $H_{0}^{1}(\Omega)$. Then, for all $w \in \mathcal{I}_{0}$ and for all $A \in \mathbb{R}$,

$$
0 \leq \lambda_{A} \leq \frac{\int_{\Omega}|\nabla w|^{2}}{\int_{\Omega} w^{2}} .
$$


Proof of Lemma 1.2. Set $\lambda_{n}=\lambda_{A_{n}}$ and $\varphi_{n}=\varphi_{A_{n}}$. Multiplying equation (0.1) by $\varphi_{A_{n}}$ and integrating over $\Omega$ yields

$$
\int\left|\nabla \varphi_{n}\right|^{2}+\frac{A_{n}}{2} \int v \cdot \nabla\left(\varphi_{n}\right)^{2}=\lambda_{n} \int\left(\varphi_{n}\right)^{2}=\lambda_{n}
$$

Since the function $\left(\varphi_{n}\right)^{2}$ is, say, in $W_{0}^{1,1}(\Omega)$ (because $\left.\varphi_{n} \in H_{0}^{1}(\Omega)\right),\left(\varphi_{n}\right)^{2}$ can be approximated in $W^{1,1}$ norm by a sequence of functions $\left(u_{k}\right)_{k \in \mathbb{N}} \in C_{0}^{\infty}(\Omega)$. Since div $v=0$ in $\mathcal{D}^{\prime}(\Omega)$, it follows that $\int v \cdot \nabla u_{k}=0$ and the passage to the limit $k \rightarrow+\infty$ gives $\int v \cdot \nabla\left(\varphi_{n}\right)^{2}=0$. Hence,

$$
\lambda_{n}=\int\left|\nabla \varphi_{n}\right|^{2}
$$

From Rellich's theorem, there exists a subsequence $n^{\prime} \rightarrow+\infty$ and a function $w \in$ $H_{0}^{1}(\Omega)$ such that $\varphi_{n^{\prime}} \rightarrow w$ strongly in $L^{2}$ and weakly in $H^{1}$. Furthermore, $\|w\|_{L^{2}(\Omega)}=1$ and (1.3) holds.

On the other hand, we also know that $\Delta \varphi_{n^{\prime}} \rightarrow \Delta w$ in the sense of distributions. Therefore, dividing (0.1) by $A_{n^{\prime}}$ and passing to the limit $n^{\prime} \rightarrow+\infty$, one gets that $v \cdot \nabla w=0$ almost everywhere in $\Omega$.

Proof of Lemma 1.3. Let $A \in \mathbb{R}$ be given and let $\varphi$ be a (positive) first eigenfunction of (0.1) for the first eigenvalue $\lambda$. We drop the subscripts $A$ for the sake of keeping notations simple. Observe first that $\lambda$ is nonnegative by (1.5) ( $\lambda$ is actually positive since $\varphi$ is not constant). Let $w$ be in $\mathcal{I}_{0}$.

Fix any $\varepsilon>0$ and multiply the equation $(0.1)$ by $w^{2} /(\varphi+\varepsilon) \in W_{0}^{1,1}(\Omega)$. One gets

$$
-\int \Delta \varphi \frac{w^{2}}{\varphi+\varepsilon}+A \int v \cdot \nabla\left(w^{2} \ln (\varphi+\varepsilon)\right)-2 A \int w \ln (\varphi+\varepsilon) v \cdot \nabla w=\lambda \int w^{2} \frac{\varphi}{\varphi+\varepsilon} .
$$

Since the function $w^{2} /(\varphi+\varepsilon)$ is in $W_{0}^{1,1}(\Omega)$, it can be approximated in $W^{1,1}$ norm by a sequence of function $\left(u_{k}\right)_{k \in \mathbb{N}} \in C_{0}^{\infty}(\Omega)$. On the other hand, by standard estimates, $\Delta \varphi \in L^{\infty}(\Omega)$, and $\nabla \varphi \in L^{\infty} \cap W^{1, p}$ (for any $1 \leq p<+\infty$ ). Therefore, the first term of the left-hand side of (1.6) can be estimated by

$$
\begin{aligned}
-\int \Delta \varphi \frac{w^{2}}{\varphi+\varepsilon} & =-\lim _{k \rightarrow+\infty} \int \Delta \varphi u_{k}=\lim _{k \rightarrow+\infty} \int \nabla \varphi \cdot \nabla u_{k} \\
& =\int \nabla \varphi \cdot \nabla\left(\frac{w^{2}}{\varphi+\varepsilon}\right)=\int \frac{2 w(\varphi+\varepsilon) \nabla \varphi \cdot \nabla w-w^{2}|\nabla \varphi|^{2}}{(\varphi+\varepsilon)^{2}} \\
& \leq \int|\nabla w|^{2}
\end{aligned}
$$

The second term of the left-hand side of (1.6) is equal to 0 since $w^{2} \ln (\varphi+\varepsilon) \in W_{0}^{1,1}$ and $v$ is divergence-free. Lastly, the third term clearly vanishes since $w$ is a first integral. 
Therefore,

$$
\lambda \int w^{2} \frac{\varphi}{\varphi+\varepsilon} \leq \int|\nabla w|^{2}
$$

and (1.4) follows from Lebesgue's dominated convergence theorem, passing to the limit as $\varepsilon \rightarrow 0^{+}$. This completes the proof of Lemma 1.3 and that of Theorem 0.3.

Remark 1.4 It follows from (1.5) that

$$
\lambda_{A} \geq \lambda_{0}=\min _{\phi \in H_{0}^{1}(\Omega),} \frac{\int_{\Omega}|\nabla \phi|^{2}}{\int_{\Omega} \phi^{2}}
$$

where $\lambda_{0}$ is the first eigenvalue of (0.1) with $A=0$. Theorem 0.3 then says that if $v$ has a first integral, the $\lambda_{A}$ 's converge to the minimum (which is achieved) of the Rayleigh quotient $\int_{\Omega}|\nabla w|^{2} / \int_{\Omega} w^{2}$ over all first integrals (instead of over all nonzero $\phi \in H_{0}^{1}(\Omega)$ for $A=0)$.

Furthermore, Lemmas 1.2 and 1.3 immediately yield the following

Corollary 1.5 If $v$ has first integrals (in $H_{0}^{1}$ ), then, for any sequence of eigenfunctions $\left(\varphi_{A_{n}}\right)$ of (0.1) with $\left\|\varphi_{A_{n}}\right\|_{L^{2}(\Omega)}=1$, there exists a subsequence $\varphi_{A_{n^{\prime}}}$ which converges weakly in $H^{1}$ and strongly in $L^{2}$ to a minimizer of the Dirichlet functional among all first integrals.

Remark 1.6 Whereas the uniqueness (up to multiplication) of the minimizers of the Rayleigh quotient among all nonzero $H_{0}^{1}$ functions is a well-known fact, the uniqueness of the minimizers of the right-hand side of $(0.2)$, i.e. the minimizers of the Rayleigh quotient among all nonzero $H_{0}^{1}$ first integrals of $v$, if any, does not hold in general.

Indeed, consider for instance, in dimension 2, the disk $\Omega=\left\{x_{1}^{2}+x_{2}^{2}<1\right\}$ and $v=\left(0, \alpha\left(x_{1}\right)\right)$, where $\alpha$ is, say, an even $C^{1}$ function such that $\alpha=0$ on $[-b,-a] \cup[a, b]$, $\alpha \neq 0$ on $[-1,-b) \cup(-a, a) \cup(b, 1]$ with $0<a<b<1$. Call $\omega^{-}=\Omega \cap\{-b<$ $\left.x_{1}<-a\right\}$ and $\omega^{+}=\Omega \cap\left\{a<x_{1}<b\right\}$, and let $\lambda$ and $\varphi$ be the first eigenvalue and eigenfunction of the Laplace operator in $\omega^{+}$with Dirichlet boundary conditions. Up to normalization, let us assume that $\varphi>0$ in $\omega^{+}$and $\|\varphi\|_{L^{2}\left(\omega^{+}\right)}=1 / 2$. Let us extend $\varphi$ by 0 in $\Omega \backslash \omega^{+}$. It is straightforward to see in this case that $v$ has first integrals and $\mathcal{I}_{0}=\left\{w \in H_{0}^{1}(\Omega) \backslash\{0\}, w=0\right.$ a.e. in $\left.\Omega \backslash\left(\omega^{+} \cup \omega^{-}\right)\right\}$. Hence

$$
\min _{w \in \mathcal{I}_{0}} \frac{\int_{\Omega}|\nabla w|^{2}}{\int_{\Omega} w^{2}}=\lambda
$$

and the set of the minimizers of the above Rayleigh quotient is the set of functions of the type $c^{+} \chi_{\omega^{+}} \varphi\left(x_{1}, x_{2}\right)+c^{-} \chi_{\omega^{-}} \varphi\left(-x_{1}, x_{2}\right)$, where $\left(c^{+}, c^{-}\right) \in \mathbb{R}^{2} \backslash\{(0,0)\}$ and $\chi$ denotes 
the characteristic function. Furthermore, since each first eigenvalue $\varphi_{A}$ of $(0.1)$ is even in $x_{1}$, one can say, assuming that $\left\|\varphi_{A}\right\|_{L^{2}(\Omega)}=1$, that the whole family $\left(\varphi_{A}\right)_{A}$ converges to the function $\chi_{\omega^{+}} \varphi\left(x_{1}, x_{2}\right)+\chi_{\omega^{-}} \varphi\left(-x_{1}, x_{2}\right)$ as $A \rightarrow+\infty$.

On the contrary, let $\Omega=\{|x|<1\}$ be the unit ball in $\mathbb{R}^{N}$ and assume that $v \in C^{1}(\bar{\Omega})$ is such that $v \cdot x=0$ in $\Omega$ and $v(x) \neq 0$ for $x \neq 0$. Then $v$ has first integrals and $\mathcal{I}_{0}$ is the set of nonzero radial functions in $H_{0}^{1}(\Omega)$. But since the Rayleigh quotient has a unique minimizer among all nonzero $H_{0}^{1}(\Omega)$ functions and since this unique minimizer is radial and is the first eigenfunction $\varphi$ of the Laplace operator in $\Omega$ with Dirichlet boundary conditions, it follows that this Rayleigh quotient has a unique minimizer in $\mathcal{I}_{0}$. Furthermore, one can say in this case that, after normalization, each $\varphi_{A}$ is equal to $\varphi$, and, for all $A, \lambda_{A}$ is the first eigenvalue associated with $\varphi$.

\section{Extensions to more general elliptic equations, and to Neumann or periodic boundary conditions}

\subsection{More general linear equations with Dirichlet boundary con- ditions}

As in section 1 , one still assumes that $\Omega$ is a $C^{2}$ bounded domain of $\mathbb{R}^{N}$ and that $v$ is a bounded vector field such that $\operatorname{div} v=0$ in $\mathcal{D}^{\prime}(\Omega)$. Let now $a(x)=\left(a_{i j}(x)\right)_{1 \leq i, j \leq N}$ be a $C^{1}(\bar{\Omega})$ symmetric matrix field such that

$$
\exists 0<\alpha \leq \beta, \forall x \in \Omega, \forall \xi \in \mathbb{R}^{N}, \quad \alpha|\xi|^{2} \leq \sum_{1 \leq i, j \leq N} a_{i j}(x) \xi_{i} \xi_{j} \leq \beta|\xi|^{2},
$$

and let $c(x)$ be a function in $L^{\infty}(\Omega)$. Lastly, let $p$ be a measurable function in $\Omega$ such that $0<p_{1} \leq p(x) \leq p_{2}$ a.e. in $\Omega$, for some positive constants $p_{1}$ and $p_{2}$.

The proof of Theorem 0.3 can easily be extended to the following more general situation :

Theorem 2.1 For all $A \in \mathbb{R}$, let $\lambda_{A}$ be the principal eigenvalue, and $\varphi_{A}$ be the principal eigenfunction (up to multiplication) of

$$
\left\{\begin{aligned}
-\operatorname{div}\left(a \nabla \varphi_{A}\right)+A v \cdot \nabla \varphi_{A}+c \varphi_{A} & =\lambda_{A} p \varphi_{A} & & \text { in } \Omega \\
\varphi_{A} & =0 & & \text { on } \partial \Omega .
\end{aligned}\right.
$$

If $v$ has a first integral in $H_{0}^{1}(\Omega)$, then

$$
\lambda_{A} \rightarrow \min _{w \in \mathcal{I}_{0}} \frac{\int_{\Omega} \nabla w \cdot a(x) \nabla w+c(x) w^{2}}{\int_{\Omega} p w^{2}} \text { as } A \rightarrow+\infty .
$$

If $v$ has no first integral in $H_{0}^{1}(\Omega)$, then $\lambda_{A} \rightarrow+\infty$ as $A \rightarrow+\infty$. 
As for Theorem 0.3, we can add furthermore that

$$
\lambda_{A} \leq \frac{\int_{\Omega} \nabla w \cdot a(x) \nabla w+c(x) w^{2}}{\int_{\Omega} p w^{2}}
$$

for all $A \in \mathbb{R}$ and for all $w \in \mathcal{I}_{0}$.

The comments and remarks in section 1 can be extended to the more general problem (2.2). Let us especially mention that one always has $\lambda_{A} \geq \operatorname{essinf}_{\Omega}(c / p)$. Furthermore, $\lambda_{A}=O\left(A^{2}\right)$ as soon as $v$ is continuous at a point in $\Omega$ with or without the divergence free assumption. Lastly, if there exists a $C^{2}(\bar{\Omega})$ field $U$ such that $v=a \nabla U$, then $\lambda_{A}$ is the principal eigenvalue, with weight $p$, of the following problem

$$
-\operatorname{div}(a \nabla \psi)+\left(\frac{A^{2}}{4}(\nabla U \cdot a \nabla U)-\frac{A}{2} \operatorname{div} v\right) \psi+c \psi=\lambda_{A} p \psi
$$

with Dirichlet boundary conditions, whence $\lambda_{A} \sim A^{2} \times \min _{\bar{\Omega}}((\nabla U \cdot a \nabla U) /(4 p))$ as $A \rightarrow+\infty$ under the additional assumption that, say, $p$ is continuous in $\bar{\Omega}$ and $v$ does not vanish in $\bar{\Omega}$.

\subsection{Neumann or periodic boundary conditions}

The case of Neumann or periodic boundary conditions is slightly different since, as we shall see below, first integrals always exist. Let us now describe what we mean by periodic and/or Neumann boundary conditions.

Let $d$ be an integer such that $0 \leq d \leq N$ and call $x=\left(x_{1}, \cdots, x_{d}\right)$ and $y=\left(x_{d+1}, \cdots, x_{N}\right)$. Let $L_{1}, \cdots, L_{d}$ be $d$ positive numbers and let $\Omega$ be a $C^{2}$ connected open subset of $\mathbb{I R}^{N}$ such that

$$
\exists R \geq 0, \quad \forall(x, y) \in \Omega, \quad|y| \leq R
$$

and

$$
\forall\left(k_{1}, \cdots, k_{d}\right) \in L_{1} \mathbb{Z} \times \cdots \times L_{d} \mathbb{Z}, \quad \Omega=\Omega+\sum_{i=1}^{d} k_{i} e_{i},
$$

where $\left(e_{i}\right)_{1 \leq i \leq N}$ is the canonical basis of $\mathbb{R}^{N}$.

Let $C$ be the set defined by

$$
C=\left\{(x, y) \in \Omega, x \in\left(0, L_{1}\right) \times \cdots \times\left(0, L_{d}\right)\right\} .
$$

In the case $d=0$, then $\Omega$ is bounded and $C=\Omega$; otherwise, $\Omega$ is unbounded and $C$ is its cell of periodicity. A function $w$ is said to be $L$-periodic with respect to $x$ in $\Omega$ if $w(x+k, y)=w(x, y)$ almost everywhere in $\Omega$ for all $k \in L_{1} \mathbb{Z} \times \cdots \times L_{d} \mathbb{Z}$. 
Let $a(x, y)=\left(a_{i j}(x, y)\right)_{1 \leq i, j \leq N}$ be a $C^{1}(\bar{\Omega})$ and $L$-periodic with respect to $x$ matrix field satisfying

$$
\exists 0<\alpha \leq \beta, \forall(x, y) \in \bar{\Omega}, \forall \xi \in \mathbb{R}^{N}, \quad \alpha|\xi|^{2} \leq \sum_{1 \leq i, j \leq N} a_{i j}(x, y) \xi_{i} \xi_{j} \leq \beta|\xi|^{2} .
$$

Let $v$ be a $L^{\infty}(\Omega)$ vector field such that $v \in W^{1,1}\left(\Omega_{b}\right)$ for all $b>0$, where $\Omega_{b}=\{(x, y) \in$ $\Omega,|x|<b\}$. Assume furthermore that $v$ is $L$-periodic with respect to $x$ and satisfies

$$
\operatorname{div} v=0 \text { a.e. in } \Omega \text { and } v \cdot \nu=0 \text { in } L_{l o c}^{1}(\partial \Omega) \text {, }
$$

where $\nu$ denotes the unit outward normal to $\Omega$. Let $c(x, y)$ be a $L^{\infty}(\Omega)$ function and assume that $c$ is $L$-periodic with respect to $x$.

Let $\mathcal{H}$ be the set of all functions $w$ which are $L$-periodic with respect to $x$ and which belong to $H^{1}\left(\Omega_{b}\right)$ for all $b>0$. A nonzero function $w \in \mathcal{H}$ is said to be a first integral of $v$ if $v \cdot \nabla w=0$ almost everywhere in $\Omega$. Let $\mathcal{I}$ be the set of all first integrals. Note that this set is not empty since it contains all nonzero constant functions.

For all $A \in \mathbb{R}$, there exists a unique principal eigenvalue $\lambda_{A}$, and a unique (up to multiplication) function $\varphi_{A}$, which is positive in $\bar{\Omega}, L$-periodic with respect to $x$, and solves

$$
\left\{\begin{aligned}
-\operatorname{div}\left(a \nabla \varphi_{A}\right)+A v \cdot \nabla \varphi_{A}+c \varphi_{A} & =\lambda_{A} \varphi_{A} & & \text { in } \Omega \\
\nu \cdot a \nabla \varphi_{A} & =0 & & \text { on } \partial \Omega .
\end{aligned}\right.
$$

Furthermore, $\varphi_{A}$ belongs to $W^{2, p}\left(\Omega_{b}\right)$ for all $b>0$ and $1 \leq p<+\infty$ (hence, $\varphi_{A} \in C^{1, \alpha}(\bar{\Omega})$ for all $0 \leq \alpha<1$ ).

This general framework includes the case of a bounded domain with Neumann type boundary conditions (case $d=0$ ), that of an infinite (straight or oscillating) cylinder with periodic coefficients and Neumann boundary conditions, as well as the case of the whole space $\mathbb{R}^{N}$ with periodic holes and/or periodic coefficients ( see also [4] for a detailed study of problem (2.7)).

Theorem 2.2 The principal eigenvalues $\lambda_{A}$ are bounded and

$$
\lambda_{A} \rightarrow \min _{w \in \mathcal{I}} \frac{\int_{C} \nabla w \cdot a(x, y) \nabla w+c(x, y) w^{2}}{\int_{C} w^{2}} \text { as } A \rightarrow+\infty .
$$

As for Theorems 0.3 and 2.1, one can say furthermore that

$$
\lambda_{A} \leq \frac{\int_{C} \nabla w \cdot a(x, y) \nabla w+c(x, y) w^{2}}{\int_{C} w^{2}}
$$

for all $A \in \mathbb{R}$ and $w \in \mathcal{I}$. 
Remark 2.3 It is clear that, unlike problems (0.1) or (2.2), the principal eigenvalues $\lambda_{A}$ of problem (2.7) are always bounded as $A \rightarrow+\infty$. Indeed, one has

$$
\forall A \in \mathbb{R}, \quad \inf _{\Omega} c \leq \lambda_{A} \leq \sup _{\Omega} c .
$$

The purpose of Theorem 2.2 is to prove that the $\lambda_{A}$ 's do converge as $A \rightarrow+\infty$ and to determine their limit.

Remark 2.4 In the case of the whole space $\mathbb{R}^{N}$, and under the additional assumption that $v$ has zero average, it is known that there exists a skewsymmetric and $L$-periodic matrix field $B$ such that $v=-\operatorname{div} B$ (see [14], [23]). In this particular case, (2.7) reduces to $-\operatorname{div}\left((a+A B) \nabla \varphi_{A}\right)+c \varphi_{A}=\lambda_{A} \varphi_{A}$. However, because of the lack of symmetry of the diffusion term, this last formulation does not seem to be helpful to derive the limit of $\lambda_{A}$ as $A \rightarrow+\infty$.

Proof of Theorem 2.2. The proof follows the main lines of that of Theorem 0.3. Let us especially mention that the integrations by parts are made over the set $C$. Notice also that the additional assumptions $v \cdot \nu=0$ in $L_{l o c}^{1}(\partial \Omega)$ and $v \in W^{1,1}\left(\Omega_{b}\right)$ (for all $b>0$ ), together with div $v=0$, are used to guarantee that the integrals of the type $\int_{C} v \cdot \nabla \varphi_{A}^{2}$ vanish (from Green's formula and the periodicity assumptions).

Let us just sketch the proof of the upper bound for $\lambda_{A}$. Choose $A \in \mathbb{R}$ and $w \in \mathcal{I}$. Since $\varphi_{A} \in C^{1}(\bar{\Omega})$ is positive on $\bar{\Omega}$, the function $w^{2} / \varphi_{A}$ is in $W^{1,1}\left(\Omega_{b}\right)$ (for all $b>0$ ), and, since $\Omega$ is $L$-periodic and at least of class $C^{1}$, the function $w^{2} / \varphi_{A}$ can be approximated in the norms of $W^{1,1}\left(\Omega_{b}\right)$ by a sequence of functions $\left(u_{k}\right)_{k} \in C^{1}(\bar{\Omega})$ which are $L$-periodic with respect to $x$. Each function $u_{k}$ is in $\mathcal{H}$, whence

$$
\int_{C} \nabla u_{k} \cdot a \nabla \varphi_{A}+A \int_{C} u_{k} v \cdot \nabla \varphi_{A}+\int_{C} c u_{k} \varphi_{A}=\lambda_{A} \int_{C} u_{k} \varphi_{A}
$$

and

$$
\int_{C} \nabla \frac{w^{2}}{\varphi_{A}} \cdot a \nabla \varphi_{A}+A \int_{C} w^{2} v \cdot \nabla \ln \varphi_{A}+\int_{C} c w^{2}=\lambda_{A} \int_{C} w^{2}
$$

after passing to the limit $k \rightarrow+\infty$. Since $w \in \mathcal{I}$, one has $w^{2} v \cdot \nabla \ln \varphi_{A}=v \cdot \nabla\left(w^{2} \ln \varphi_{A}\right)$. The function $w^{2} \ln \varphi_{A}$ is in $W^{1,1}\left(\Omega_{b}\right)$ (for all $b>0$ ) and $L$-periodic with respect to $x$. It can then be approximated in the norms of $W^{1,1}\left(\Omega_{b}\right)$ by a sequence of functions $\left(U_{k}\right)_{k} \in C^{1}(\bar{\Omega})$ which are $L$-periodic with respect to $x$. Therefore, using Green's formula and (2.6), one gets

$$
\int_{C} v \cdot \nabla\left(w^{2} \ln \varphi_{A}\right)=\lim _{k \rightarrow+\infty} \int_{C} v \cdot \nabla U_{k}=\lim _{k \rightarrow+\infty} \int_{C} U_{k} \operatorname{div} v=0 .
$$

One then concludes as in Lemma 1.3 that

$$
\lambda_{A} \leq \frac{\int_{C} \nabla w \cdot a \nabla w+c w^{2}}{\int_{C} w^{2}}
$$


and the proof of Theorem 2.2 is complete.

Remark 2.5 (On the necessity of the assumption $v \cdot \nu=0$ on $\partial \Omega$ ) Unlike problems (0.1) or (2.2) with Dirichlet boundary conditions, the additional assumption stating that $v \cdot \nu=0$ on $\partial \Omega$ is needed to determine the limit of the principal eigenvalues $\lambda_{A}$ of (2.7). Otherwise, formula (2.8) may or may not hold in general.

Consider for instance the eigenvalue problem

$$
\left\{\begin{aligned}
-\varphi_{A}^{\prime \prime}+A \varphi_{A}^{\prime}+c(x) \varphi_{A} & =\lambda_{A} \varphi_{A} \text { in }(0,1) \\
\varphi_{A}^{\prime}(0)=\varphi_{A}^{\prime}(1) & =0 .
\end{aligned}\right.
$$

The velocity field $v=1$ does not satisfy the assumption $v \cdot \nu=0$ at 0 and 1 . The first integrals of $v$ are the nonzero constants. If $c \equiv 0$, then (2.8) is clearly true since $\lambda_{A}=0$ for all $A \in \mathbb{R}$. On the other hand, for general $c$, after rewriting problem (2.9) in a self-adjoint way :

$$
\left\{\begin{aligned}
-\left(e^{-A x} \varphi_{A}^{\prime}\right)^{\prime}+c(x) e^{-A x} \varphi_{A} & =\lambda_{A} e^{-A x} \varphi_{A} \quad \text { in }(0,1) \\
\varphi_{A}^{\prime}(0)=\varphi_{A}^{\prime}(1) & =0
\end{aligned}\right.
$$

it follows that

$$
\lambda_{A}=\min _{\phi \in H^{1}(0,1)} \frac{\int_{0}^{1} e^{-A x}\left(\phi^{\prime}\right)^{2}+c(x) e^{-A x} \phi^{2}}{\int_{0}^{1} e^{-A x} \phi^{2}} \leq \frac{\int_{0}^{1} c(x) e^{-A x}}{\int_{0}^{1} e^{-A x}} .
$$

Hence, $\lim \sup _{A \rightarrow+\infty} \lambda_{A} \leq c(0)$. On the other hand, since the first integrals to the vector field $v=1$ are the nonzero constants, the right hand side of (2.8) is here nothing else but $\int_{0}^{1} c(x) d x$. Therefore, (2.8) does not hold for problem (2.9) as soon as $c(0)<\int_{0}^{1} c(x) d x$.

\section{Applications to parabolic equations}

The results of section 1 can be applied to characterize the behavior in finite time of the solutions of Cauchy problems with large advection. Namely, let $\Omega$ be a $C^{2}$ bounded domain in $\mathbb{R}^{N}$, let $v$ be a $L^{\infty}(\Omega)$ and satisfying $\operatorname{div} v=0$ in $\mathcal{D}^{\prime}(\Omega)$. For any $u_{0} \in H_{0}^{1}(\Omega)$ and $A \in \mathbb{R}$, let $u^{A}=u^{A}(t, \cdot)$ be the solution of

$$
\left\{\begin{aligned}
u_{t}^{A} & =\Delta u^{A}-A v \cdot \nabla u^{A}, \quad t>0 \\
u^{A}(t, \cdot) & =0 \text { on } \partial \Omega, \quad t \geq 0 \\
u^{A}(0, \cdot) & =u_{0}
\end{aligned}\right.
$$

This solution $u^{A}$ belongs to $C\left(\mathbb{R}^{+}, H_{0}^{1}\right) \cap L^{2}\left(0, T ; H_{0}^{1} \cap H^{2}\right)$, and $u_{t}^{A} \in L^{2}\left(0, T ; L^{2}\right)$ for all $T>0$. 
Theorem 3.1 The following properties are equivalent:

(i) there exists $u_{0} \in H_{0}^{1}(\Omega)$ such that $u^{A}(1, \cdot) \nrightarrow 0$ in $L^{2}(\Omega)$ as $A \rightarrow+\infty$,

(ii) the vector field $v$ has a nonzero first integral $w \in H_{0}^{1}(\Omega)$,

(iii) the first eigenvalues $\lambda_{A}$ of (0.1) are bounded as $A \rightarrow+\infty$.

One has already proved in Theorem 0.3 that properties (ii) and (iii) are equivalent. Theorem 3.1 says further that the nonexistence of first integrals is equivalent to the fact that the solutions of (3.1), with any fixed initial datum, go to 0 in $L^{2}$ in finite time (see also Remark 3.2 below) as the amplitude of the drift goes to $+\infty$.

In the case of Hamiltonian systems in even dimensions, where $v$ is given as the orthogonal gradient of a first integral $H, v=\bar{\nabla} H$, the behavior of the functions $u^{A}$ can be made more precise (see Fannjiang, Papanicolaou [14], Wentzell and Freidlin [38]). But, as far as we know, the characterization of the decay of the functions $u^{A}$ in finite time in terms of the first integrals of $v$, in the general case of non-Hamiltonian systems, had not been investigated yet.

Remark 3.2 (On the behavior of the solutions of (3.1) at other finite times or other times scales) Theorem 3.1 also holds if time $t=1$ in property (i) is replaced with any positive time $t=T$, and even if property (i) is replaced with $\min _{t_{1} \leq t \leq t_{2}}\left\|u^{A}(t, \cdot)\right\|_{L^{2}(\Omega)} \nrightarrow 0$ as $A \rightarrow+\infty$, given any two positive times $0<t_{1} \leq t_{2}$. Indeed, as it can easily be seen by multiplying (3.1) by $u^{A}$, each function $t \mapsto\left\|u^{A}(t, \cdot)\right\|_{L^{2}(\Omega)}$ is nonincreasing (see the proof of Theorem 3.1 below).

On the other hand, any solution $u^{A}$ of (3.1) with $A>0$ gives rise to a solution $U^{A}(t, \cdot)=u^{A}(t / A, \cdot)$ of

$$
\left\{\begin{aligned}
U_{t}^{A} & =A^{-1} \Delta U^{A}-v \cdot \nabla U^{A}, \quad t>0 \\
U^{A}(t, \cdot) & =0 \text { on } \partial \Omega, \quad t \geq 0 \\
U^{A}(0, \cdot) & =u_{0}
\end{aligned}\right.
$$

The limiting behavior of the functions $U^{A}$ in finite time -which corresponds to times proportional to $1 / A$ for the solutions of problem (3.1)- has been thoroughly studied (see [14] and [38]). The above Theorem 3.1 then gives some information about the behavior of the functions $U^{A}$ for times proportional to $A$.

Proof of Theorem 3.1. As already emphasized, assertions (ii) and (iii) are equivalent, from Theorem 0.3.

Proof of ((ii) and/or (iii)) $\Longrightarrow$ (i). From the proof of Theorem 0.3, there exists a sequence $A_{n} \rightarrow+\infty$ and a nonzero first integral $w \in H_{0}^{1}(\Omega)$ such that the first eigenfunctions $\varphi_{n}=\varphi_{A_{n}}$ of problem (0.1), normalized so that $\left\|\varphi_{n}\right\|_{L^{2}(\Omega)}=1$, converge to $w$ weakly in $H_{0}^{1}$ and strongly in $L^{2}$.

Take the initial condition $u_{0}=w$ for the solutions $u_{n}:=u^{A_{n}}$ of problem (3.1), and call

$$
h_{n}(t, \cdot):=u_{n}(t, \cdot)-v_{n}(t, \cdot)
$$


where $v_{n}(t, \cdot)=e^{-\lambda_{A_{n}} t} \varphi_{n}(\cdot)$. Since each function $v_{n}$ solves (3.1) with $A=A_{n}$ and initial condition $\varphi_{n}$, it follows that $h_{n}$ is a solution of

$$
\left\{\begin{aligned}
\left(h_{n}\right)_{t} & =\Delta h_{n}-A_{n} v \cdot \nabla h_{n}, \quad t>0 \\
h_{n}(t, \cdot) & =0 \text { on } \partial \Omega, \quad t \geq 0 \\
h_{n}(0, \cdot) & =w-\varphi_{n}
\end{aligned}\right.
$$

Multiplying the above equation by $h_{n}(t, \cdot)$ and integrating by parts over $\Omega$ and $\left[t_{1}, t_{2}\right]$ leads to

$$
\begin{aligned}
\frac{1}{2}\left\|h_{n}\left(t_{2}, \cdot\right)\right\|_{L^{2}(\Omega)}^{2}-\frac{1}{2}\left\|h_{n}\left(t_{1}, \cdot\right)\right\|_{L^{2}(\Omega)}^{2} & =-\int_{t_{1}}^{t_{2}}\left(\int_{\Omega}\left|\nabla h_{n}(t, x)\right|^{2} d x\right) d t \\
& \leq 0
\end{aligned}
$$

for all $0 \leq t_{1} \leq t_{2}$. Therefore,

$$
\left\|h_{n}(1, \cdot)\right\|_{L^{2}(\Omega)} \leq\left\|h_{n}(0, \cdot)\right\|_{L^{2}(\Omega)}=\left\|w-\varphi_{n}\right\|_{L^{2}(\Omega)} .
$$

Since the right-hand side of the above inequality goes to 0 as $n \rightarrow+\infty$, it follows from Theorem 0.3 that $u_{n}(1, \cdot) \rightarrow e^{-\lambda_{\infty}} w \neq 0$ in $L^{2}(\Omega)$, where $\lambda_{\infty}=\min _{w \in \mathcal{I}_{0}} \int_{\Omega}|\nabla w|^{2} / \int_{\Omega} w^{2}$.

Proof of (i) $\Longrightarrow\left(\left(\right.\right.$ ii) and/or (iii)). Let $u_{0} \in H_{0}^{1}(\Omega) \backslash\{0\}, \varepsilon>0$ and $A_{n} \rightarrow+\infty$ such that

$$
\forall n \in \mathbb{N}, \quad\left\|u_{n}(1, \cdot)\right\|_{L^{2}(\Omega)} \geq \varepsilon
$$

where $u_{n}=u^{A_{n}}$.

Call $u_{n, \pm}$ the solution of problem (3.1) with $A=A_{n}$ and initial condition $u_{0}^{ \pm} \in H_{0}^{1}(\Omega)$, where $u_{0}^{ \pm}=\chi_{\left\{ \pm u_{0}>0\right\}} u_{0}$. By linearity and uniqueness, one has $u_{n}=u_{n,+}+u_{n,-}$. On the other hand, the maximum principle implies that, for all $t \geq 0, u_{n,+}(t, x) \geq 0$ and $u_{n,-}(t, x) \leq 0$ almost everywhere in $\Omega$. Lastly, one has either $\left\|u_{n,+}(1, \cdot)\right\|_{L^{2}(\Omega)} \geq \varepsilon / 2$ or $\left\|u_{n,-}(1, \cdot)\right\|_{L^{2}(\Omega)} \geq \varepsilon / 2$. Therefore, up to extraction of some subsequence, and even if it means changing $\varepsilon / 2$ into $\varepsilon$, one can assume without loss of generality that (3.3) holds with $u_{0} \geq 0$ a.e. in $\Omega$ (and hence, for all $t \geq 0, u_{n}(t, \cdot) \geq 0$ a.e. in $\Omega$ ).

Call now $u_{0}^{M}=\chi_{\left\{u_{0}<M\right\}} u_{0}$ and $M>0$ large enough so that $\left\|u_{0}-u_{0}^{M}\right\|_{L^{2}(\Omega)} \leq \varepsilon / 2$. Let $u_{n}^{M}$ and $v_{n}$ be the solutions of (3.1) with initial conditions $u_{0}^{M}$ and $u_{0}-u_{0}^{M} \in H_{0}^{1}(\Omega)$. One has $u_{n}=u_{n}^{M}+v_{n}$ and, as in (3.2), the function $t \mapsto\left\|v_{n}(t, \cdot)\right\|_{L^{2}(\Omega)}$ is nonincreasing, whence $\left\|v_{n}(1, \cdot)\right\|_{L^{2}(\Omega)} \leq\left\|v_{n}(0, \cdot)\right\|_{L^{2}(\Omega)}=\left\|u_{0}-u_{0}^{M}\right\|_{L^{2}(\Omega)} \leq \varepsilon / 2$. Thus, $\left\|u_{n}^{M}(1, \cdot)\right\|_{L^{2}(\Omega)} \geq \varepsilon / 2$. On the other hand, it follows from the maximum principle that, for all $t \geq 0, u_{n}^{M}(t, \cdot) \leq M$ a.e. in $\Omega$. Therefore, even if it means changing $\varepsilon / 2$ into $\varepsilon$, one can assume without loss of generality that (3.3) holds with $0 \leq u_{0} \leq M$ a.e. in $\Omega$ (and hence, for all $t \geq 0$, $0 \leq u_{n}(t, \cdot) \leq M$ a.e. in $\Omega$ ).

Since the function $t \mapsto\left\|u_{n}(t, \cdot)\right\|_{L^{2}(\Omega)}$ is continuous and nonincreasing, it follows that

$$
\forall n \in \mathbb{N}, \quad\left\|u_{n}\right\|_{L^{2}((0,1) \times \Omega)} \leq\left\|u_{0}\right\|_{L^{2}(\Omega)} .
$$


Up to extraction of some subsequence, one can then assume that $u_{n} \rightarrow w$ weakly in $L^{2}((0,1) \times \Omega)$.

Furthermore, it also follows as in (3.2) that $\nabla u_{n}$ is uniformly bounded in $L^{2}((0,1) \times$ $\Omega)$, namely

$$
\iint_{(0,1) \times \Omega}\left|\nabla u_{n}\right|^{2} \leq \frac{1}{2}\left\|u_{0}\right\|_{L^{2}(\Omega)}^{2} .
$$

Therefore, standard arguments give that $\frac{\partial w}{\partial x_{i}} \in L^{2}((0,1) \times \Omega)$ for all $1 \leq i \leq N$. Fubini's theorem then yields that $w(t, \cdot) \in H^{1}(\Omega)$ for almost every $t \in(0,1)$.

Fix now any $i \in\{1, \cdots, N\}$ and any function $\varphi(t, x)$ of class $C^{1}$ and with compact support in $(0,1) \times \mathbb{R}^{N}$. For all $n \in \mathbb{N}$ and $t \in(0,1)$, the function $u_{n}(t, \cdot)$ is in $H_{0}^{1}(\Omega)$ and $\varphi(t, \cdot) \in C^{1}\left(\mathbb{R}^{N}\right)$ with compact support. Hence

$$
\left|\int_{\Omega} u_{n}(t, x) \frac{\partial \varphi}{\partial x_{i}}(t, x) d x\right| \leq\left\|\frac{\partial u_{n}}{\partial x_{i}}(t, \cdot)\right\|_{L^{2}(\Omega)}\|\varphi(t, \cdot)\|_{L^{2}(\Omega)}
$$

and it follows from Cauchy-Schwarz inequality that

$$
\begin{aligned}
\left|\iint_{(0,1) \times \Omega} u_{n} \frac{\partial \varphi}{\partial x_{i}}\right| & \leq \int_{0}^{1}\left\|\frac{\partial u_{n}}{\partial x_{i}}(t, \cdot)\right\|_{L^{2}(\Omega)}\|\varphi(t, \cdot)\|_{L^{2}(\Omega)} d t \\
& \leq\left\|\nabla u_{n}\right\|_{L^{2}((0,1) \times \Omega)}\|\varphi\|_{L^{2}((0,1) \times \Omega)} .
\end{aligned}
$$

Therefore, one gets from (3.4) that

$$
\left|\iint_{(0,1) \times \Omega} w \frac{\partial \varphi}{\partial x_{i}}\right|=\left|\lim _{n \rightarrow+\infty} \iint_{(0,1) \times \Omega} u_{n} \frac{\partial \varphi}{\partial x_{i}}\right| \leq C\|\varphi\|_{L^{2}((0,1) \times \Omega)}
$$

where $C=\left\|u_{0}\right\|_{L^{2}(\Omega)} / \sqrt{2}$ is independent of $\varphi$. One then concludes that $w(t, \cdot) \in H_{0}^{1}(\Omega)$ for almost every $t \in(0,1)$.

On the other hand, the functions $\left(u_{n}\right)_{t}$ (resp. $\Delta u_{n}$ ) converge to $w_{t}$ (resp. $\Delta w$ ) in $\mathcal{D}^{\prime}((0,1) \times \Omega)$. By dividing by $A_{n}$ the equation (3.1) satisfied by $u_{n}$ and by passing to the limit $n \rightarrow+\infty$, it follows that $v \cdot \nabla w=0$ almost everywhere in $(0,1) \times \Omega$. Hence, for almost every $t \in(0,1)$, the function $v(\cdot) \cdot \nabla w(t, \cdot) \in L^{1}(\Omega)$ and is equal to 0 a.e. in $\Omega$.

Lastly, since $0 \leq u_{n} \leq M$ and since the function $t \mapsto\left\|u_{n}(t, \cdot)\right\|_{L^{2}(\Omega)}$ is nonincreasing, it is found that

$$
M|\Omega| \geq \iint_{(0,1) \times \Omega} u_{n} \geq \frac{1}{M} \iint_{(0,1) \times \Omega} u_{n}^{2} \geq \frac{1}{M}\left\|u_{n}(1, \cdot)\right\|_{L^{2}(\Omega)}^{2},
$$

where $|\Omega|$ denotes the Lebesgue measure of $\Omega$. Thus,

$$
M|\Omega| \geq \iint_{(0,1) \times \Omega} u_{n} \geq \frac{\varepsilon^{2}}{M}
$$


from (3.3). Since $u_{n} \rightarrow w$ weakly in $L^{2}((0,1) \times \Omega)$ and since $(0,1) \times \Omega$ is bounded, one gets that $\iint_{(0,1) \times \Omega} u_{n} \rightarrow \iint_{(0,1) \times \Omega} w$ as $n \rightarrow+\infty$, whence

$$
M|\Omega| \geq \iint_{(0,1) \times \Omega} w \geq \frac{\varepsilon^{2}}{M}>0 .
$$

To sum up, one knows that, for almost every $t \in(0,1)$, the function $w(t, \cdot)$ is in $H_{0}^{1}(\Omega)$ and satisfies $v(\cdot) \cdot \nabla w(t, \cdot)=0$ almost everywhere in $\Omega$. From (3.5), one eventually concludes that there exists at least a $t \in(0,1)$ such that $w(t, \cdot)$ is a nonzero first integral of $v$ in $H_{0}^{1}(\Omega)$.

That completes the proof of Theorem 3.1.

Remark 3.3 Under the same notations as in section 2.1, it is immediate to check that Theorem 3.1 also holds if equation (3.1) is replaced with the more general parabolic problem

$$
u_{t}^{A}=\operatorname{div}\left(a \nabla u^{A}\right)-A v \cdot \nabla u^{A}+c u^{A},
$$

where $c \in L^{\infty}(\Omega)$ and $a$ is a $C^{1}(\bar{\Omega})$ symmetric matrix field satisfying (2.1).

\section{Applications to nonlinear propagation phenomena}

The previous sections dealt with the asymptotic behavior of some solutions of linear elliptic or parabolic equations with high first-order coefficients. This behavior is directly related to the first integrals of the underlying velocity field $v$.

This section is concerned with nonlinear propagation phenomena for some reactiondiffusion-advection equations in periodic domains. We study the asymptotic behavior of the speeds of propagation of pulsating travelling fronts in the limit of large advection coefficients, and, as in the previous sections, we will shed light on the role played by the first integrals of the velocity field.

We consider here the general framework described in section 2.2. Let $\Omega$ be a domain satisfying (2.3-2.4) and assume here that $d \geq 1$. The latter implies that $\Omega$ is unbounded in the $d$ variables $\left(x_{1}, \cdots, x_{d}\right)$. Let $v=\left(v_{1}, \cdots, v_{N}\right)$ be an $L$-periodic with respect to $x$ and $C^{1, \delta}(\bar{\Omega})$ (with $\delta>0$ ) vector field satisfying (2.6) and

$$
\forall 1 \leq i \leq d, \quad \int_{C} v_{i} d x d y=0
$$

Let $a(x, y)=\left(a_{i j}(x, y)\right)_{1 \leq i, j \leq N}$ be a symmetric, $L$-periodic with respect to $x$, and $C^{3}(\bar{\Omega})$ matrix field satisfying $(2.5)$. 
Lastly, let $f(x, y, u)$ be a nonnegative function defined in $\bar{\Omega} \times[0,1]$ and such that

$$
\left\{\begin{array}{l}
f \text { is } L \text {-periodic with respect to } x, \\
f \text { is globally Lipschitz-continuous and } \exists \delta>0, f \text { is } C^{1, \delta} \text { with respect to } u, \\
\forall(x, y) \in \bar{\Omega}, \quad f(x, y, 0)=f(x, y, 1)=0, \\
\exists \rho \in(0,1), \quad \forall(x, y) \in \bar{\Omega}, \quad \forall 1-\rho \leq s \leq s^{\prime} \leq 1, \quad f(x, y, s) \geq f\left(x, y, s^{\prime}\right), \\
\forall s \in(0,1), \quad \exists(x, y) \in \bar{\Omega}, \quad f(x, y, s)>0, \\
\forall(x, y) \in \bar{\Omega}, \quad f_{u}^{\prime}(x, y, 0):=\lim _{u \rightarrow 0^{+}} f(x, y, u) / u>0 .
\end{array}\right.
$$

The simplest case of a function $f(x, y, u)$ satisfying $(4.2)$ is when $f(x, y, u)=\tilde{f}(u)$ and the $C^{1, \delta}$ function $\tilde{f}$ satisfies : $\tilde{f}(0)=\tilde{f}(1)=0, \tilde{f}>0$ on $(0,1), \tilde{f}^{\prime}(0)>0$ and $\tilde{f}^{\prime}(1)<0$. Such nonlinearities arise especially in combustion and biological models [1], [15], [31]. Another example of such a function $f$ is $f(x, y, u)=h(x, y) \tilde{f}(u)$ where $\tilde{f}$ is as before and $h$ is $L$-periodic with respect to $x$, Lipschitz-continuous and positive in $\bar{\Omega}$ (see [33] for such an example in ecology).

We are interested with the propagation of fronts in the domain $\Omega$, with the diffusion $a$, the advection $A v$ and the reaction $f$, and we want to analyze the asymptotic behavior of their speeds of propagation in the limit $A \rightarrow+\infty:$ more precisely, for any given unit vector $e=\left(e^{1}, \cdots, e^{d}\right)$ in $\mathbb{R}^{d}$, a function $u(t, x, y)$, defined for all $t \in \mathbb{R}$ and $(x, y) \in \bar{\Omega}$, satisfying $0 \leq u \leq 1$ and solving

$$
\left\{\begin{aligned}
\frac{\partial u}{\partial t}-\operatorname{div}(a \nabla u)+A v \cdot \nabla u & =f(x, y, u), \quad t \in \mathbb{R},(x, y) \in \Omega, \\
\nu \cdot a \nabla u & =0, \quad t \in \mathbb{R},(x, y) \in \partial \Omega
\end{aligned}\right.
$$

is called a pulsating travelling front propagating in direction $e$ with a so-called effective speed $c \neq 0$ if it satisfies

$$
\left\{\begin{aligned}
& \forall k \in \prod_{i=1}^{d} L_{i} \mathbb{Z}, \forall(t, x, y) \in \mathbb{R} \times \bar{\Omega}, \quad u\left(t-\frac{k \cdot e}{c}, x, y\right)=u(t, x+k, y), \\
& u(t, x, y) \underset{x \cdot e \rightarrow+\infty}{\longrightarrow} 0, \quad u(t, x, y) \underset{x \cdot e \rightarrow-\infty}{\longrightarrow} 1,
\end{aligned}\right.
$$

where the above limits hold locally in $t$ and uniformly in $y$ and in the directions of $\mathbb{R}^{d}$ orthogonal to $e$. Call $\tilde{e}$ the vector defined by $\tilde{e}=\left(e^{1}, \cdots, e^{d}, 0, \cdots, 0\right) \in \mathbb{R}^{N}$.

Under the above assumptions, it was proved in [4] that there exists $c_{A}^{*}(e)>0 \mathrm{such}$ that pulsating travelling fronts $u$ in the direction $e$ with the speed $c$ exist if and only if $c \geq c_{A}^{*}(e)$ (other results with more general nonlinearities $f$ were proved in [4], see Remark 4.2 below).

Theorem 4.1 Let e be a unit direction in $\mathbb{R}^{d}$. Under the above assumptions and under the notations of section 2.2, one has:

a) if there is a first integral $w \in \mathcal{I}$ such that $\int_{C}(v \cdot \tilde{e}) w^{2}>0$, then

$$
0<\gamma \leq \liminf _{A \rightarrow+\infty} \frac{c_{A}^{*}(e)}{A} \leq \limsup _{A \rightarrow+\infty} \frac{c_{A}^{*}(e)}{A} \leq \delta
$$


where

$$
\begin{gathered}
0<\gamma=\max \left(\sup _{w \in \mathcal{I}^{1}} \frac{\int_{C}(v \cdot \tilde{e}) w^{2}}{\int_{C} w^{2}}, \sup _{w \in \mathcal{I}^{2}} \frac{m \int_{C}(v \cdot \tilde{e}) w^{2}}{m \int_{C} w^{2}+\int_{C} \nabla w \cdot a \nabla w-\int_{C} \zeta w^{2}}\right), \\
\zeta(x, y)=f_{u}^{\prime}(x, y, 0), m=|C|^{-1} \int_{C} \zeta, \\
\mathcal{I}^{1}=\left\{w \in \mathcal{I}, \int_{C} \zeta w^{2} \geq \int_{C} \nabla w \cdot a \nabla w>0\right\}, \\
\mathcal{I}^{2}=\left\{w \in \mathcal{I}, 0<\int_{C} \zeta w^{2}<\int_{C} \nabla w \cdot a \nabla w\right\},
\end{gathered}
$$

and

$$
0<\delta=\sup _{w \in \mathcal{I}} \frac{\int_{C}(v \cdot \tilde{e}) w^{2}}{\int_{C} w^{2}},
$$

b) if all first integrals $w \in \mathcal{I}$ satisfy $\int_{C}(v \cdot \tilde{e}) w^{2} \leq 0$, then $c_{A}^{*}(e)=o(A)$ as $A \rightarrow+\infty$.

Remark 4.2 (General positive nonlinearities and combustion-type nonlinearities) Since the necessary and sufficient condition for the minimal speed to have a lower bound which is linear with respect to the amplitude of the flow only depends on the flow $v$ itself, the results of Theorem 4.1 can be used to deduce some facts for other nonlinearities $f$. Consider for instance either the case of a general nonnegative nonlinearity $f$ satisfying the first five assumptions in (4.2), but maybe not the last one, or, second, let $f$ be a nonnegative function satisfying

$$
\left\{\begin{array}{l}
f \text { is } L \text {-periodic with respect to } x, \\
f \text { is globally Lipschitz-continuous and } \exists \delta>0, \quad f \text { is } C^{1, \delta} \text { with respect to } u, \\
\exists \theta \in(0,1), \quad \forall(x, y) \in \bar{\Omega}, \quad \forall s \in[0, \theta] \cup\{1\}, \quad f(x, y, s)=0, \\
\exists \rho \in(0,1-\theta), \forall(x, y) \in \bar{\Omega}, \forall 1-\rho \leq s \leq s^{\prime} \leq 1, \quad f(x, y, s) \geq f\left(x, y, s^{\prime}\right), \\
\forall s \in(\theta, 1), \quad \exists(x, y) \in \bar{\Omega}, \quad f(x, y, s)>0 .
\end{array}\right.
$$

It is known (see [4]) that, under the above notations, there still exists a minimal speed $c_{A}^{*}(e)>0$ in the first case, whereas there is a unique speed $c_{A}(e)>0$ and a unique (up to shift in time) pulsating travelling front solving (4.3-4.4) if $f$ satisfies (4.6). In both cases, one has $c_{A}^{*}(e)$ (resp. $\left.c_{A}(e)\right) \leq \bar{c}_{A}^{*}(e)$, where $\bar{c}_{A}^{*}(e)$ is the minimal speed associated with a nonlinearity $\bar{f}$ satisfying (4.2) and such that $\bar{f} \geq f$. Therefore, $c_{A}^{*}(e)\left(\operatorname{resp} . c_{A}(e)\right)$ $=O(A)$ and

$$
0 \leq \limsup _{A \rightarrow+\infty} \frac{c_{A}^{*}(e)}{A}\left(\operatorname{resp} . \limsup _{A \rightarrow \infty} \frac{c_{A}(e)}{A}\right) \leq \sup _{w \in \mathcal{I}} \int_{C}(v \cdot \tilde{e}) w^{2} / \int_{C} w^{2}
$$


where the latter is zero if there is no first integral $w \in \mathcal{I}$ such that $\int_{C}(v \cdot \tilde{e}) w^{2}>0$ (indeed, the choice $w=1$ gives $\int_{C}(v \cdot \tilde{e}) w^{2}=0$ because of $(4.1)$ ). Furthermore,

$$
\limsup _{A \rightarrow+\infty} \frac{c_{A}^{*}(e)}{A}\left(\operatorname{resp} . \limsup _{A \rightarrow+\infty} \frac{c_{A}(e)}{A}\right) \leq \max _{(x, y) \in \bar{\Omega}} v(x, y) \cdot \tilde{e}
$$

Many papers have been devoted to the study of travelling fronts for reaction-diffusion equations of the type (4.3) since the pioneering paper by Kolmogorov, Petrovsky and Piskunov [31] in the one-dimensional case, for the equation $u_{t}=u_{x x}+f(u)$. Other results for other nonlinear functions $f$, including the bistable case, were obtained in [1], [15], [29]. Periodic nonlinearities $f(x, u)$ in space dimension 1 were first considered by Shigesada, Kawasaki and Teramoto [33], and by Hudson and Zinner [28]. The case of shear flows $v=(\alpha(y), 0, \cdots, 0)$ in straight infinite cylinders $\Omega=\mathbb{R} \times \omega$ was dealt with by Berestycki, Larrouturou, Lions [6], and Berestycki and Nirenberg [7]. The case of the whole space $\mathbb{R}^{N}$ with periodic diffusion and advection was considered by Xin [35] for a combustion-type nonlinearity $f$ (for which the speed of propagation of fronts is unique). The homogenization limit in $\mathbb{R}^{N}$ with coefficients having small periods was investigated by Fannjiang, Papanicolau [14], Freidlin [18], Heinze [23], Majda and Souganidis [32], and Xin [36]. Heinze also considered the case of the whole space with small periodic holes [24]. Formulas for the unique or minimal speeds of propagation of fronts were obtained by Hamel [22] and Heinze, Papanicolaou and Stevens [26] (similar formulas for systems of one-dimensional equations had been proved by Volpert, Volpert and Volpert [34]).

Let us temporarily come back to the case of shear flows

$$
v=\left(\alpha\left(x_{2}, \cdots, x_{N}\right), 0, \cdots, 0\right)
$$

in straight infinite cylinders $\Omega=\mathbb{R} \times \omega=\left\{\left(x_{1}, x^{\prime}\right), x_{1} \in \mathbb{R}, x^{\prime}=\left(x_{2}, \cdots, x_{N}\right) \in \omega\right\}$, where the section $\omega$ may or may not be bounded (under our general assumptions, the boundedness of $\omega$ simply means that $d=1$ ). Assume that the diffusion $a$ and the reaction $f$ are independent of $x_{1}$. Therefore, under the general notations of this section, $L_{1}$ can be any arbitrary positive number and the travelling fronts in direction $\pm e_{1}$ can be written as $u(t, x)=\phi\left(x_{1}+c t, x^{\prime}\right)$. Without loss of generality, assume furthermore that $\alpha$ is not constant and has zero average (over $\omega$ if $d=1$ or over the cell of periodicity if $d>1$ ). Under these conditions, several lower and upper bounds for the speeds of such fronts were derived by Audoly, Berestycki and Pomeau [2], Constantin, Kiselev and Ryzhik [12], [30] and Heinze [25] for combustion-type or general positive nonlinearities. Furthermore, Berestycki [3] proved that, if the function $f=f(u)$ satisfies (4.2) and the additional assumption $f(u) \leq f^{\prime}(0) u$ for all $u \in[0,1]$, then 1$) c_{A}^{*}\left( \pm e_{1}\right)$ is increasing with 
$A,^{2}$ 2) $c_{A}^{*}\left( \pm e_{1}\right) / A$ is decreasing with $A$ and

$$
\exists \beta>0, \quad c_{A}^{*}\left( \pm e_{1}\right) / A \rightarrow \beta>0 \text { as } A \rightarrow+\infty .
$$

The latter is more precise than the results of Theorem 4.1 in that case. Such an exact linear behavior is unknown for the general periodic setting, as well as for a function $f=f(u)$ satisfying only (4.2), or (4.6), even in the case of shear flows. However, one can deduce from Theorem 4.1 that, for a shear flow $v=\left(\alpha\left(x^{\prime}\right), 0, \cdots, 0\right)$ with nonconstant $\alpha$ having zero average, one has

$$
\liminf _{A \rightarrow+\infty} \frac{c_{A}^{*}(e)}{A}>0
$$

for any function $f$ satisfying (4.2) and for any unit vector $e \in \mathbb{R}^{d}$ such that $\tilde{e} \cdot e_{1} \neq 0$, the diffusion $a$ and the reaction $f$ maybe depending (periodically) on $x_{1}$ with period $L_{1}$ (a more precise lower bound in (4.9) can also be derived from Theorem 4.1). Formula (4.9) follows from the observation that any nonzero function $w=w\left(x_{1}, x^{\prime}\right)=w\left(x^{\prime}\right)$ which is in $\mathcal{H}$ is a first integral ; therefore, there are first integrals $w \in \mathcal{I}$ such that $\int_{C}(v \cdot \tilde{e}) w^{2}>0$ ( see also the proof of Corollary 4.3 below).

Recently, Heinze [25] showed that, in the case of shear flows in infinite cylinders, without dependance on $x_{1}$ in the coefficients of (4.3) and with $f=f(u)$ satisfying (4.2), inequality (4.8) for $c_{A}^{*}\left( \pm e_{1}\right)$ may be strict if, for instance, $f^{\prime}(0)$ is small enough. Audoly, Berestycki and Pomeau [2] formally derived the asymptotics $c_{A}^{*}\left( \pm e_{1}\right) \sim$ $A \max _{x^{\prime} \in \bar{\omega}}\left( \pm \alpha\left(x^{\prime}\right)\right)$ in the limit of high reaction (together with high advection). Under the above assumptions, the latter was made rigorous by Constantin, Kiselev and Ryzhik [12]. That can also be viewed as an immediate consequence of Theorem 4.1, in a more general periodic setting and with the direction of propagation which may not be that of the flow :

Corollary 4.3 Under the assumptions of Theorem 4.1, call $c_{A, B}^{*}(e)$ the minimal speed of pulsating travelling fronts solving (4.3-4.4) with nonlinearity $B f$ instead of $f$, for $B>0$. Then, for any $\varepsilon>0$, there exists $B_{0}>0$ such that, for all $B \geq B_{0}$,

$$
\sup _{w \in \mathcal{I}} \frac{\int_{C}(v \cdot \tilde{e}) w^{2}}{\int_{C} w^{2}}-\varepsilon \leq \liminf _{A \rightarrow+\infty} \frac{c_{A, B}^{*}(e)}{A} \leq \limsup _{A \rightarrow+\infty} \frac{c_{A, B}^{*}(e)}{A} \leq \sup _{w \in \mathcal{I}} \frac{\int_{C}(v \cdot \tilde{e}) w^{2}}{\int_{C} w^{2}} .
$$

In particular, under the above assumptions, let $v=\left(\alpha\left(x^{\prime}\right), 0, \cdots, 0\right)$ be a non-constant shear flow with zero average, in an infinite cylinder $\Omega=\mathbb{R} \times \omega$, where $x^{\prime}=\left(x_{2}, \cdots, x_{N}\right)$, where $\omega$ may or may not be bounded, and where $a$ and $f$ depend (periodically) on $x_{1}$ with period $L_{1}$. Then, for any $\varepsilon>0$, there exists $B_{0}>0$ such that, for all $B \geq B_{0}$,

$$
\max _{x^{\prime} \in \bar{\omega}}\left(\tilde{e} \cdot e_{1} \alpha\left(x^{\prime}\right)\right)-\varepsilon \leq \liminf _{A \rightarrow+\infty} \frac{c_{A, B}^{*}(e)}{A} \leq \limsup _{A \rightarrow+\infty} \frac{c_{A, B}^{*}(e)}{A} \leq \max _{x^{\prime} \in \bar{\omega}}\left(\tilde{e} \cdot e_{1} \alpha\left(x^{\prime}\right)\right) \text {. }
$$

\footnotetext{
${ }^{2}$ One here makes a slight abuse of notation by calling $c_{A}^{*}\left( \pm e_{1}\right)$ the minimal speed of the travelling fronts propagating in the direction $\pm x_{1}$.
} 
The proof of this corollary is given at the end of this section.

Another special class of flows are the rotating flows. Consider, say, a two-dimensional rotating flow of the type $v=\left(-\partial_{y} \psi, \partial_{x} \psi\right)$, where $\psi(x, y)=\sin (x) \sin (y)$. Under the above notations, formal arguments by Audoly, Berestycki and Pomeau [2] lead to an asymptotic behavior proportional to $c_{A}^{*}\left(e_{1}\right) \sim \beta A^{1 / 4}$ as $A \rightarrow+\infty$, for some $\beta>0$. The estimate $c_{A}^{*}\left(e_{1}\right) \geq \kappa A^{1 / 5}$, for some positive constant $\kappa$, was obtained by Kiselev and Ryzhik [30] (these estimates actually hold for the bulk burning rate - see [11]- which concerns more generally speaking the solutions of the Cauchy problem (4.3) in infinite cylinders and coincides with the speed of propagation for travelling fronts, if any).

Theorem 4.1 applied to this case gives the following additional information that, in any direction $e$, the minimal speed can not grow like $A$ :

Corollary 4.4 Let $v=\left(-\partial_{y} \psi, \partial_{x} \psi\right)$ be a two-dimensional rotating flow where, say, $\psi(x, y)=\sin (x) \sin (y)$. Then, under the notations of Theorem 4.1, $c_{A}^{*}(e)=o(A)$ as $A \rightarrow+\infty$, for any direction e of $\mathbb{R}^{2}$.

Proof. For such a flow $v$, it is easy to see that, for any direction $e$ and any first integral $w \in \mathcal{I}$, then $\int_{(0,2 \pi)^{2}}(v \cdot e) w^{2}=0$. The conclusion follows then from Theorem 4.1.

Before going into the proof of Theorem 4.1, let us lastly point out another consequence of Theorem 4.1, which deals with the case of small diffusion and bounded (from above and below) advection and reaction :

Corollary 4.5 Under the notations at the beginning of this section, let $\gamma_{\varepsilon}^{*}(e)>0$ be the minimal speed of propagation of pulsating fronts u solving (4.4) and

$$
\left\{\begin{aligned}
\frac{\partial u}{\partial t}-\varepsilon \operatorname{div}(a \nabla u)+v \cdot \nabla u & =f(x, y, u), \quad t \in \mathbb{R},(x, y) \in \Omega, \\
\nu \cdot a \nabla u & =0, \quad t \in \mathbb{R},(x, y) \in \partial \Omega,
\end{aligned}\right.
$$

a) Then

$$
\liminf _{\varepsilon \rightarrow 0^{+}} \gamma_{\varepsilon}^{*}(e) \geq \sup _{w \in \mathcal{I}} \frac{\int_{C}(v \cdot \tilde{e}) w^{2}}{\int_{C} w^{2}} .
$$

b) Furthermore, if $v$ is a shear flow $v=\left(\alpha\left(x^{\prime}\right), 0, \cdots, 0\right)$ with zero average, in an infinite cylinder $\Omega=\mathbb{R} \times \omega$, where $x^{\prime}=\left(x_{2}, \cdots, x_{N}\right)$ and $\omega$ may or may not be bounded, if $a_{i 1}=0$ for $i \geq 2$, if $a_{11}$ is constant and if $a$ and $f$ only depend on $x^{\prime}$, then

$$
\gamma_{\varepsilon}^{*}\left( \pm e_{1}\right) \rightarrow \max _{x^{\prime} \in \bar{\omega}}\left( \pm \alpha\left(x^{\prime}\right)\right) \quad \text { as } \varepsilon \rightarrow 0^{+}
$$

Corollary 4.5 is proved at the end of this section. 
Remark 4.6 Other asymptotics have been considered in the literature. Many works have for instance dealt with the solutions of Cauchy problems for equations of the type (4.12), with small diffusion $\varepsilon$, together with large reaction $\varepsilon^{-1} f$. Typically, the solutions of such Cauchy problems converge as $\varepsilon \rightarrow 0^{+}$to two-phase solutions of Hamilton-Jacobi type equations, separated by interfaces : see Freidlin [19] and Majda and Souganidis [32], where other spatio-temporal scales and various homogenization limits have also been considered.

Let us now turn to the

Proof of Theorem 4.1. Let us begin with the

Proof of the lower bound of a). Let $e$ be a unit direction of $\mathbb{R}^{d}$ and choose any first integral $w \in \mathcal{I}$ such that

$$
\int_{C}(v \cdot \tilde{e}) w^{2}>0
$$

We shall now estimate the minimal speed $c_{A}^{*}(e)$ from below for large $A$.

Remember that $\zeta$ is the function defined in $\bar{\Omega}$ by $\zeta(x, y)=f_{u}^{\prime}(x, y, 0)$. It follows from [4] and [5] that

$$
c_{A}^{*}(e) \geq \min _{\lambda>0} \frac{\mu(\lambda)}{\lambda}
$$

where $\mu(\lambda)$ is the principal eigenvalue of the operator

$$
L_{\lambda} \psi:=\operatorname{div}(a \nabla \psi)-\lambda[\operatorname{div}(a \tilde{e} \psi)+\tilde{e} \cdot a \nabla \psi]-A v \cdot \nabla \psi+\left(\lambda A v \cdot \tilde{e}+\lambda^{2} \tilde{e} \cdot a \tilde{e}+\zeta\right) \psi
$$

acting on the set $E_{\lambda}$ of functions $\psi(x, y)$ which are $L$-periodic with respect to $x$ in $\Omega$ and satisfy $\nu \cdot a(-\tilde{e} \lambda \psi+\nabla \psi)=0$ on $\partial \Omega .^{3}$

The right-hand side of (4.14) only depends on $e$, the domain $\Omega$, the coefficients $A v$ and $a$, and the dependence on $f$ is only through the $f_{u}^{\prime}(\cdot, \cdot, 0)$. Let us also mention that the equality holds in (4.14) under the additional assumption $f(x, y, u) \leq f_{u}^{\prime}(x, y, 0) u$ for all $u \in[0,1]$ and for all $(x, y) \in \bar{\Omega}($ see $[5])$. In the case where $\Omega=\mathbb{R}^{N}, a=I, v=0$ and $f=f(u)$ satisfies $(4.2)$ with $f(u) \leq f^{\prime}(0) u$ in $\left.[0,1]\right)$, the latter reduces to the well-known KPP formula $c_{A}^{*}(e)=2 \sqrt{f^{\prime}(0)}$ for the minimal speed of planar fronts [31].

Fix any positive $\lambda$ and call $\psi$ the unique (up to multiplication) positive (in $\bar{\Omega}$ ) solution $\psi \in E_{\lambda}$ of

$$
L_{\lambda} \psi=\mu(\lambda) \psi
$$

First, divide (4.15) by $\psi$ and integrate over the cell $C$. Using (2.6) and the boundary conditions satisfied by $\psi$, it follows that

$$
\int_{C} \frac{\nabla \psi \cdot a \nabla \psi}{\psi^{2}}-2 \lambda \frac{\tilde{e} \cdot a \nabla \psi}{\psi}+\lambda^{2} \tilde{e} \cdot a \tilde{e}+\zeta=\mu(\lambda)|C| .
$$

\footnotetext{
${ }^{3}$ Such operators $L_{\lambda}$ also arise in Bloch eigenvalue problems (see [9], [10]).
} 
Therefore,

$$
\mu(\lambda)|C|=\int_{C}\left(\frac{\nabla \psi}{\psi}-\lambda \tilde{e}\right) \cdot a\left(\frac{\nabla \psi}{\psi}-\lambda \tilde{e}\right)+\zeta
$$

whence

$$
\mu(\lambda) \geq m=|C|^{-1} \int_{C} \zeta
$$

because of $(2.5)$.

Then, multiply (4.15) by $w^{2} / \psi$, where $w \in \mathcal{I}$ is a first integral such that $\int_{C}(v \cdot \tilde{e}) w^{2}>$ 0 . Observe that the term

$$
\int_{C} A v \cdot \frac{\nabla \psi}{\psi} w^{2}
$$

vanishes after integration by parts since $v \cdot \nabla w=0$, since $v, \psi$ and $w$ are $L$-periodic with respect to $x$, and because of (2.6). Therefore,

$$
\begin{aligned}
\mu(\lambda) \int_{C} w^{2}= & \int_{C} \frac{\nabla \psi \cdot a \nabla \psi}{\psi^{2}} w^{2}-2 \frac{w \nabla w \cdot a \nabla \psi}{\psi}-2 \lambda w^{2} \frac{\tilde{e} \cdot a \nabla \psi}{\psi} \\
& +\int_{C} 2 \lambda w \tilde{e} \cdot a \nabla w+\lambda^{2} w^{2} \tilde{e} \cdot a \tilde{e}+\zeta w^{2}+\lambda A(v \cdot \tilde{e}) w^{2} .
\end{aligned}
$$

For any $t \in(0,1)$, the first three terms in the right-hand side of (4.17) can be estimated as follows:

$$
\begin{gathered}
\int_{C} \frac{\nabla \psi \cdot a \nabla \psi}{\psi^{2}} w^{2}-2 \frac{w \nabla w \cdot a \nabla \psi}{\psi}-2 \lambda w^{2} \frac{\tilde{e} \cdot a \nabla \psi}{\psi} \\
=\int_{C}\left(t \frac{\nabla \psi \cdot a \nabla \psi}{\psi^{2}} w^{2}-2 \frac{w \nabla w \cdot a \nabla \psi}{\psi}\right)+\int_{C}\left((1-t) \frac{\nabla \psi \cdot a \nabla \psi}{\psi^{2}} w^{2}-2 \lambda w^{2} \frac{\tilde{e} \cdot a \nabla \psi}{\psi}\right) \\
\geq-\int_{C} \frac{1}{t} \nabla w \cdot a \nabla w+\frac{1}{1-t} \lambda^{2} w^{2} \tilde{e} \cdot a \tilde{e}
\end{gathered}
$$

because of (2.5). Putting the above inequality into (4.17) leads to

$$
\begin{aligned}
\mu(\lambda) \int_{C} w^{2} \geq & -\int_{C}\left(\frac{1}{t} \nabla w \cdot a \nabla w+\frac{t}{1-t} \lambda^{2} w^{2} \tilde{e} \cdot a \tilde{e}\right) \\
& +\int_{C} 2 \lambda w \tilde{e} \cdot a \nabla w+\zeta w^{2}+\lambda A(v \cdot \tilde{e}) w^{2} .
\end{aligned}
$$

Let us set

$$
\gamma=\int_{C} \nabla w \cdot a \nabla w>0 \text { and } \eta=\int_{C} w^{2} \tilde{e} \cdot a \tilde{e}>0
$$

Maximizing the right-hand side of (4.18) over all $t \in(0,1)$ gives, with $t=$ $(1+\lambda \sqrt{\eta / \gamma})^{-1}$

$$
\begin{aligned}
\mu(\lambda) \int_{C} w^{2} \geq & \int_{C}-\left(1+\lambda \sqrt{\frac{\eta}{\gamma}}\right) \nabla w \cdot a \nabla w-\lambda \sqrt{\frac{\gamma}{\eta}} w^{2} \tilde{e} \cdot a \tilde{e}+2 \lambda w \tilde{e} \cdot a \nabla w \\
& +\int_{C} \zeta w^{2}+\lambda A(v \cdot \tilde{e}) w^{2} \\
= & \int_{C}\left(\zeta w^{2}-\nabla w \cdot a \nabla w\right)-\lambda \sqrt{\frac{\gamma}{\eta}} \int_{C} z \cdot a z+\lambda A \int_{C}(v \cdot \tilde{e}) w^{2} .
\end{aligned}
$$


where $z=\sqrt{\frac{\eta}{\gamma}} \nabla w-w \tilde{e}$.

Let us now consider two cases, according to the sign of $\int_{C} \zeta w^{2}-\nabla w \cdot a \nabla w$.

Case 1: $\int_{C} \zeta w^{2} \geq \int_{C} \nabla w \cdot a \nabla w>0$. From (4.14) and (4.19), it follows that

$$
c_{A}^{*}(e) \geq \min _{\lambda>0} \frac{\mu(\lambda)}{\lambda} \geq-\sqrt{\frac{\gamma}{\eta}} \frac{\int_{C} z \cdot a z}{\int_{C} w^{2}}+A \frac{\int_{C}(v \cdot \tilde{e}) w^{2}}{\int_{C} w^{2}} .
$$

Therefore,

in case 1.

$$
\liminf _{A \rightarrow+\infty} \frac{c_{A}^{*}(e)}{A} \geq \frac{\int_{C}(v \cdot \tilde{e}) w^{2}}{\int_{C} w^{2}}
$$
$\lambda>0$,

Case 2 : $0<\int_{C} \zeta w^{2}<\int_{C} \nabla w \cdot a \nabla w$. It follows from (4.16) and (4.19) that, for any

$$
\frac{\mu(\lambda)}{\lambda} \geq \max \left(\frac{m}{\lambda}, h(\lambda)\right)
$$

where

$$
h(\lambda)=\frac{1}{\int_{C} w^{2}}\left(\frac{1}{\lambda} \int_{C}\left(\zeta w^{2}-\nabla w \cdot a \nabla w\right)-\sqrt{\frac{\gamma}{\eta}} \int_{C} z \cdot a z+A \int_{C}(v \cdot \tilde{e}) w^{2}\right) .
$$

The functions $\lambda \mapsto m / \lambda$ and $h$ are respectively decreasing and increasing, and, for $A$ large enough, their graphs have exactly one intersection point, which is the minimum over all positive $\lambda$ of the function $\lambda \mapsto \max (m / \lambda, h(\lambda))$. Since $c_{A}^{*}(e) \geq \min _{\lambda>0} \mu(\lambda) / \lambda$, a straightforward calculation gives

$$
\liminf _{A \rightarrow+\infty} \frac{c_{A}^{*}(e)}{A} \geq \frac{m \int_{C}(v \cdot \tilde{e}) w^{2}}{m \int_{C} w^{2}+\int_{C} \nabla w \cdot a \nabla w-\int_{C} \zeta w^{2}}
$$

in case 2 .

Putting cases 1 and 2 together completes the proof of the lower bound in part a) of Theorem 4.1.

Let us now turn to the

Proof of the upper bound in a) and proof of part b) of Theorem 4.1. Fix a direction $e$ of $\mathbb{R}^{d}$ and assume that $\limsup _{A \rightarrow+\infty} c_{A}^{*}(e) / A>0$ and choose any positive number $\kappa$ such that

$$
0<\kappa<\limsup _{A \rightarrow+\infty} \frac{c_{A}^{*}(e)}{A} .
$$


Let $g=g(u)$ be a function satisfying (4.2), and such that $f(x, y, u) \leq g(u)$ for all $(x, y, u) \in \bar{\Omega} \times[0,1]$ and $g(u) \leq g^{\prime}(0) u$ for all $u \in[0,1]$. Call $\gamma_{A}^{*}(e)$ the minimal speed of pulsating travelling fronts solving (4.3-4.4) with the nonlinearity $g$ instead of $f$. Let $\chi: \mathbb{R} \rightarrow \mathbb{R}$ be a smooth nondecreasing function such that $\chi(s)=0$ for all $s \leq 1$, $0<\chi(s)<1$ for all $s \in(1,2)$ and $\chi(s)=1$ for all $s \geq 2$. For each $\theta \in(0,1 / 2)$, the function $f_{\theta}(x, y, s)=\chi(s / \theta) f(x, y, s)$ is of the type (4.6). It was proved in [4] that, for each $\theta \in(0,1 / 2)$, there exists a unique speed $c_{A, \theta}(e)$ and a unique (up to shift in time) pulsating travelling front solving (4.3-4.4) with the nonlinearity $f_{\theta}$. Furthermore, $c_{A, \theta}(e) \rightarrow c_{A}^{*}(e)$ as $\theta \rightarrow 0^{+}$.

Similarly, there exists a unique speed $\gamma_{A, \theta}(e)$ and a unique (up to shift in time) pulsating travelling front solving (4.3-4.4) with the nonlinearity $g_{\theta}(s)=\chi(s / \theta) g(s)$; furthermore, $\gamma_{A, \theta}(e) \rightarrow \gamma_{A}^{*}(e)$ as $\theta \rightarrow 0^{+}$. But the results in sections 3 and 4 in [4] yield $c_{A, \theta}(e) \leq \gamma_{A, \theta}(e)$ for each $\theta \in(0,1 / 2)$ (since $f_{\theta} \leq g_{\theta}$ and both $f_{\theta}$ and $g_{\theta}$ satisfy (4.6)). Therefore, $c_{A}^{*}(e) \leq \gamma_{A}^{*}(e)$.

From (4.20), there exists then a sequence $A_{n} \rightarrow+\infty$ such that

$$
\gamma_{A_{n}}^{*}(e) \geq \kappa A_{n}
$$

for all $n \in \mathbb{N}$.

On the other hand, since $g$ satisfies (4.2) and $g(s) \leq g^{\prime}(0) s$ for all $s \in[0,1]$, it follows from [5] (as already noticed at the beginning of the proof of Theorem 4.1) that, for all $A \in \mathbb{R}$,

$$
\gamma_{A}^{*}(e)=\min _{\lambda>0} \frac{k_{A}(\lambda)}{\lambda}
$$

where $k_{A}(\lambda)$ is the principal eigenvalue of the elliptic operator

$$
\begin{aligned}
\mathcal{L}_{A, \lambda} \psi:= & \operatorname{div}(a \nabla \psi)-\lambda[\operatorname{div}(a \tilde{e} \psi)+\tilde{e} \cdot a \nabla \psi]-A v \cdot \nabla \psi \\
& +\left(\lambda A v \cdot \tilde{e}+\lambda^{2} \tilde{e} \cdot a \tilde{e}+g^{\prime}(0)\right) \psi
\end{aligned}
$$

acting on the set $E_{\lambda}$ of functions $\psi(x, y)$ which are $L$-periodic with respect to $x$ in $\Omega$ and satisfy $\nu \cdot a(-\tilde{e} \lambda \psi+\nabla \psi)=0$ on $\partial \Omega$.

From (4.21), it resorts that

$$
\forall \lambda>0, \forall n \in \mathbb{N}, \quad k_{A_{n}}(\lambda) \geq \kappa \lambda A_{n} .
$$

Fix any $\varepsilon>0$. Take $\lambda_{n}=\left(\varepsilon A_{n}\right)^{-1}$ in the above inequality and call $\psi_{n} \in E_{\lambda_{n}}$ the principal eigenfunction of

$$
\begin{aligned}
\mathcal{L}_{A_{n}, \lambda_{n}} \psi_{n}= & \operatorname{div}\left(a \nabla \psi_{n}\right)-\left(\varepsilon A_{n}\right)^{-1}\left[\operatorname{div}\left(a \tilde{e} \psi_{n}\right)+\tilde{e} \cdot a \nabla \psi_{n}\right]-A_{n} v \cdot \nabla \psi_{n} \\
& +\left(\varepsilon^{-1} v \cdot \tilde{e}+\left(\varepsilon A_{n}\right)^{-2} \tilde{e} \cdot a \tilde{e}+g^{\prime}(0)\right) \psi_{n} \\
= & k_{A_{n}}\left(\lambda_{n}\right) \psi_{n}
\end{aligned}
$$

such that $\left\|\psi_{n}\right\|_{L^{2}(C)}=1$. 
Multiply the above equality by $\psi_{n}$ and integrate by parts over $C$. One obtains

$$
\begin{aligned}
\int_{C}-\nabla \psi_{n} \cdot a \nabla \psi_{n}+\left[\varepsilon^{-1} v \cdot \tilde{e}+\left(\varepsilon A_{n}\right)^{-2} \tilde{e} \cdot a \tilde{e}+g^{\prime}(0)\right] \psi_{n}^{2} & =\int_{C} k_{A_{n}}\left(\lambda_{n}\right) \psi_{n}^{2} \\
& \geq \frac{\kappa}{\varepsilon} \int_{C} \psi_{n}^{2}
\end{aligned}
$$

from (4.22) and due to the definition of $\lambda_{n}$. Since $\int_{C} \psi_{n}^{2}=1$ and since the matrix $a$ is uniformly elliptic, one concludes that the sequence $\left\|\psi_{n}\right\|_{H^{1}(C)}$ is bounded. It also follows that the sequence $k_{A_{n}}\left(\lambda_{n}\right)$ is bounded (from above and below by two positive constants).

From Rellich's theorem, there exists then a subsequence $n^{\prime} \rightarrow+\infty$ and a function $w_{\varepsilon} \in H_{l o c}^{1}(\Omega)$ such that $\psi_{n^{\prime}}$ converges to $w_{\varepsilon}$ weakly in $H_{l o c}^{1}$, strongly in $L_{l o c}^{2}$, and almost everywhere in $\Omega$. The function $w_{\varepsilon}$ is then $L$-periodic with respect to $x$, and it is not the zero function since $\left\|w_{\varepsilon}\right\|_{L^{2}(C)}=1$.

Multiply (4.23) by $1 / A_{n^{\prime}}$ and pass to the limit in the sense of distributions in $\Omega$. It follows that $v \cdot \nabla w_{\varepsilon}=0$ almost everywhere in $\Omega$. That means that $w_{\varepsilon}$ is a (nonzero) first integral of $v$.

Passing to the limit $n^{\prime} \rightarrow \infty$ in (4.24) immediately leads to

$$
\left(\frac{\kappa}{\varepsilon}-g^{\prime}(0)\right) \int_{C} w_{\varepsilon}^{2} \leq \frac{1}{\varepsilon} \int_{C}(v \cdot \tilde{e}) w_{\varepsilon}^{2}
$$

Therefore, choosing $\varepsilon$ smaller than $\kappa / g^{\prime}(0)$ proves the existence of a first integral $w_{\varepsilon} \in \mathcal{I}$ such that $\int_{C}(v \cdot \tilde{e}) w_{\varepsilon}^{2}>0$ and

$$
\kappa \leq \frac{\int_{C}(v \cdot \tilde{e}) w_{\varepsilon}^{2}}{\int_{C} w_{\varepsilon}^{2}}+\varepsilon g^{\prime}(0) \leq \sup _{w \in \mathcal{I}} \frac{\int_{C}(v \cdot \tilde{e}) w^{2}}{\int_{C} w^{2}}+\varepsilon g^{\prime}(0) .
$$

That already proves part b) of Theorem 4.1. Furthermore, the passages to the limit $\varepsilon \rightarrow 0^{+}$and then $\kappa \stackrel{<}{\rightarrow} \limsup _{A \rightarrow+\infty} c_{A}^{*}(e) / A$ lead to

$$
\limsup _{A \rightarrow+\infty} \frac{c_{A}^{*}(e)}{A} \leq \sup _{w \in \mathcal{I}} \frac{\int_{C}(v \cdot \tilde{e}) w^{2}}{\int_{C} w^{2}}
$$

That completes the proof of Theorem 4.1.

Let us now turn to the

Proof of Corollary 4.3. The upper bound in (4.10) follows from Theorem 4.1 and (4.7), which holds whether or not there exists $w \in \mathcal{I}$ such that $\int_{C}(v \cdot \tilde{e}) w^{2}>0$. 
The lower bound in (4.10) is immediate if all first integrals $w \in \mathcal{I}$ are such that $\int_{C}(v \cdot \tilde{e}) w^{2} \leq 0$, since $c_{A, B}^{*}(e)>0$ for all $A$ and $B>0$. In the other case, let any $\varepsilon>0$ and choose a first integral $w_{0}$ in $\mathcal{I}$ such that $\int_{C}(v \cdot \tilde{e}) w_{0}^{2}>0$ and

$$
\sup _{w \in \mathcal{I}} \frac{\int_{C}(v \cdot \tilde{e}) w^{2}}{\int_{C} w^{2}}-\varepsilon \leq \frac{\int_{C}(v \cdot \tilde{e}) w_{0}^{2}}{\int_{C} w_{0}^{2}}
$$

Let $\zeta(x, y)=f_{u}^{\prime}(x, y, 0)$. Since $\zeta$ is continuous and positive in $\bar{\Omega}$, there exists $B_{0}$ such that, for all $B \geq B_{0}$,

$$
\int_{C} B \zeta w_{0}^{2} \geq \int_{C} \nabla w_{0} \cdot a \nabla w_{0}
$$

Therefore, for all $B \geq B_{0}$, under the notations of Theorem 4.1, $w_{0}$ belongs to the set $\mathcal{I}^{1}$ associated to the nonlinearity $B f$. Hence,

$$
\forall B \geq B_{0}, \quad \liminf _{A \rightarrow+\infty} \frac{c_{A, B}^{*}(e)}{A} \geq \frac{\int_{C}(v \cdot \tilde{e}) w_{0}^{2}}{\int_{C} w_{0}^{2}} \geq \sup _{w \in \mathcal{I}} \frac{\int_{C}(v \cdot \tilde{e}) w^{2}}{\int_{C} w^{2}}-\varepsilon .
$$

To prove (4.11) in the case where $v=\left(\alpha\left(x^{\prime}\right), 0, \cdots, 0\right)$, it is enough to prove that

$$
\sup _{w \in \mathcal{I}} \frac{\int_{C}(v \cdot \tilde{e}) w^{2}}{\int_{C} w^{2}}=\max _{x^{\prime} \in \bar{\omega}}\left(\tilde{e} \cdot e_{1} \alpha\left(x^{\prime}\right)\right) .
$$

The left-hand side is clearly less than or equal to the right-hand side. On the other hand, fix any $\varepsilon>0$ and choose an open set $\mathcal{U} \subset \bar{\omega}$ such that

$$
\forall x^{\prime} \in \mathcal{U}, \quad \tilde{e} \cdot e_{1} \alpha\left(x^{\prime}\right) \geq \max _{\bar{\omega}}\left(\tilde{e} \cdot e_{1} \alpha\right)-\varepsilon .
$$

Then take a smooth nonzero function $w_{0}=w_{0}\left(x^{\prime}\right)$ in $\mathcal{H}$ whose support is such that

$$
\operatorname{supp}\left(w_{0}\right) \subset \bigcup_{k \in L_{2}} \bigcup_{\mathbb{Z} \times \cdots \times L_{d}} \mathbb{Z}
$$

One can immediately see that $w_{0}$ is a first integral and

$$
\sup _{w \in \mathcal{I}} \frac{\int_{C}(v \cdot \tilde{e}) w^{2}}{\int_{C} w^{2}} \geq \frac{\int_{C}(v \cdot \tilde{e}) w_{0}^{2}}{\int_{C} w_{0}^{2}} \geq \max _{x^{\prime} \in \bar{\omega}}\left(\tilde{e} \cdot e_{1} \alpha\left(x^{\prime}\right)\right)-\varepsilon .
$$

Since $\varepsilon>0$ was arbitrary, one gets the desired result. 
Proof of Corollary 4.5. Proof of a). Let us first observe that any solution $u(t, x, y)$ of (4.12) and (4.4) with the speed $c$ gives rise to a solution $w(t, x, y):=u(t / \varepsilon, x, y)$ of

$$
\left\{\begin{aligned}
\frac{\partial w}{\partial t}-\operatorname{div}(a \nabla w)+\frac{v}{\varepsilon} \cdot \nabla w & =\frac{1}{\varepsilon} f(x, y, w), \quad t \in \mathbb{R}, \quad(x, y) \in \Omega \\
\nu \cdot a \nabla w & =0, \quad t \in \mathbb{R}, \quad(x, y) \in \partial \Omega
\end{aligned}\right.
$$

satisfying (4.4) with the speed $c / \varepsilon$. Therefore, under the notations of Corollary 4.3, one has

$$
\gamma_{\varepsilon}^{*}(e)=\varepsilon c_{\varepsilon^{-1}, \varepsilon^{-1}}^{*}(e),
$$

where $c_{\varepsilon^{-1}, \varepsilon^{-1}}^{*}(e)$ is the minimal speed of pulsating travelling fronts solving (4.3-4.4) with $A=1 / \varepsilon$ and with the nonlinearity $f / \varepsilon$ instead of $f$.

If all first integrals $w \in \mathcal{I}$ are such that $\int_{C}(v \cdot \tilde{e}) w^{2} \leq 0$, then (4.13) is immediate since $\gamma_{\varepsilon}^{*}(e)$ is positive for any $\varepsilon>0$.

Otherwise, for any $\delta>0$ small enough, there exists a first integral $w_{0} \in \mathcal{I}$ such that

$$
\frac{\int_{C}(v \cdot \tilde{e}) w_{0}^{2}}{\int_{C} w_{0}^{2}} \geq \sup _{w \in \mathcal{I}} \frac{\int_{C}(v \cdot \tilde{e}) w^{2}}{\int_{C} w^{2}}-\delta>0 .
$$

Take $M$ large enough so that

$$
\int_{C} M \zeta w_{0}^{2} \geq \int_{C} \nabla w_{0} \cdot a \nabla w_{0}
$$

Theorem 4.1 yields that

$$
\liminf _{\varepsilon \rightarrow 0^{+}} \frac{c_{\varepsilon^{-1}, M}^{*}(e)}{\varepsilon^{-1}} \geq \frac{\int_{C}(v \cdot \tilde{e}) w_{0}^{2}}{\int_{C} w_{0}^{2}} \geq \sup _{w \in \mathcal{I}} \frac{\int_{C}(v \cdot \tilde{e}) w^{2}}{\int_{C} w^{2}}-\delta
$$

On the other hand, as in the proof of Theorem 4.1, it follows that the minimal speed $c^{*}$ is nondecreasing with respect to the nonlinearity $f$. Therefore, $c_{\varepsilon^{-1}, \varepsilon^{-1}}^{*}(e) \geq c_{\varepsilon^{-1}, M}^{*}(e)$ for $\varepsilon$ small enough. Putting that together with (4.25) and (4.26) leads to

$$
\liminf _{\varepsilon \rightarrow 0^{+}} \gamma_{\varepsilon}^{*}(e) \geq \sup _{w \in \mathcal{I}} \frac{\int_{C}(v \cdot \tilde{e}) w^{2}}{\int_{C} w^{2}}-\delta
$$

Since $\delta>0$ was an arbitrary small enough positive number, the inequality (4.13) follows.

Proof of b). Let us deal with the case of the propagation in the $e_{1}$-direction (the propagation in the $-e_{1}$-direction can be treated similarly). As already observed in the 
proof of Corollary 4.3, the following formula

$$
\sup _{w \in \mathcal{I}} \frac{\int_{C}\left(v \cdot e_{1}\right) w^{2}}{\int_{C} w^{2}}=\max _{x^{\prime} \in \bar{w}} \alpha\left(x^{\prime}\right)
$$

holds for a shear flow $v=\alpha\left(x^{\prime}\right) e_{1}$.

In order to get the upper bound for $\gamma_{\varepsilon}^{*}(e)$, let us use a formula derived in [22] :

$$
\gamma_{\varepsilon}^{*}\left(e_{1}\right)=\min _{w \in \mathcal{E}} \sup _{\left(x_{1}, x^{\prime}\right) \in \bar{\Omega}}\left(-\frac{\varepsilon \operatorname{div}\left(a\left(x^{\prime}\right) \nabla w\right)+f\left(x^{\prime}, w\right)}{\partial_{x_{1}} w}+\alpha\left(x^{\prime}\right)\right),
$$

where $\mathcal{E}=\left\{w \in C^{2}(\bar{\Omega}), w\right.$ is periodic with respect to $\left(x_{2}, \cdots, x_{d}\right)$ with the periods $L_{2}, \cdots, L_{d}, \partial_{\nu} w=0$ on $\partial \Omega, \partial_{x_{1}} w<0$ in $\bar{\Omega}$ and $w\left(-\infty, x^{\prime}\right)=1, w\left(+\infty, x^{\prime}\right)=0$ uniformly in $\left.x^{\prime} \in \bar{\omega}\right\}$. Actually the above formula was proved in [22] in the case of a diffusion matrix $a=I d$, with a nonlinearity $f$ not depending on $x^{\prime}$ and in an infinite cylinder with bounded section $\omega$. The generalization to our case with diffusion and reaction depending on $x^{\prime}$ with bounded or unbounded section $\omega$ is immediate from the proof in $[22]$.

Since $a_{i 1}=0$ for $i \geq 2$ and since $a_{11}$ is constant, if follows that

$$
\gamma_{\varepsilon}^{*}\left(e_{1}\right) \leq \min _{w=w\left(x_{1}\right) \in \mathcal{E}} \sup _{x_{1} \in \mathbb{R}}\left(-\frac{\varepsilon a_{11} w^{\prime \prime}\left(x_{1}\right)+g\left(w\left(x_{1}\right)\right)}{w^{\prime}\left(x_{1}\right)}\right)+\max _{x^{\prime} \in \bar{\omega}} \alpha\left(x^{\prime}\right),
$$

where $g$ is a given function satisfying 4.2 and such that $g(u) \geq f\left(x^{\prime}, u\right)$ for all $\left(x^{\prime}, u\right) \in$ $\bar{\omega} \times[0,1]$. In other words, from $[22]$,

$$
\gamma_{\varepsilon}^{*}\left(e_{1}\right) \leq k_{\varepsilon}^{*}+\max _{x^{\prime} \in \bar{\omega}} \alpha\left(x^{\prime}\right)
$$

where $k_{\varepsilon}^{*}$ is the minimal speed of planar travelling fronts $u\left(t, x_{1}\right)=\phi\left(x_{1}-c t\right)$ solving

$$
u_{t}=\varepsilon a_{11} u_{x_{1} x_{1}}+g(u)
$$

with $\phi(-\infty)=1$ and $\phi(+\infty)=0$. It is immediate to check that $k_{\varepsilon}^{*}=\sqrt{\varepsilon} k_{1}^{*}$. Eventually, one concludes that

$$
\limsup _{\varepsilon \rightarrow 0^{+}} \gamma_{\varepsilon}^{*}\left(e_{1}\right) \leq \max _{x^{\prime} \in \bar{\omega}} \alpha\left(x^{\prime}\right) .
$$

That completes the proof of Corollary 4.5.

\section{Discussion and open questions}

The aim of this section is to set a list of open questions and generalizations of the results of the previous sections. 
Theorem 0.3 gives a necessary and sufficient condition for the first eigenvalues $\lambda_{A}$ of problem (0.1), with a divergence-free vector field $v$, be bounded as $A \rightarrow+\infty$. Moreover, the limit of $\lambda_{A}$ as $A \rightarrow+\infty$, which always exists, is either finite or equal to $+\infty$. In both cases, this limit is not smaller than any of the $\lambda_{A}$ 's. On the other hand, (1.5) implies that, for all $A \in \mathbb{R}, \lambda_{A} \geq \lambda_{0}$, where $\lambda_{0}$ corresponds to (0.1) with $A=0$ (in other words $\lambda_{0}$ is the first eigenvalue of the Laplace operator with Dirichlet boundary conditions). Furthermore, one can observe from (1.2) that $\lambda_{A}$ is a nondecreasing function of $|A|$ as soon as $v$ is a divergence-free gradient field, and it is increasing under the additional assumption that $v$ is not identically equal to 0 . However, this monotonicity property remains open for a general divergence-free vector field $v$.

As far as the first eigenfunctions $\varphi_{A}$ are concerned, it followed from the proof of Theorem 0.3 (see Corollary 1.5) that, if the field $v$ has first integrals, then each sequence $\left(\varphi_{A_{n}}\right)_{n \in \mathbb{N}}$ of normalized eigenfunctions has at least a subsequence $\left(\varphi_{A_{n^{\prime}}}\right)_{n^{\prime}}$ which converges as $n^{\prime} \rightarrow+\infty$ to a minimizer of the Rayleigh quotient among all first integrals of $v$ in $\mathcal{I}_{0}$. If this minimizer is unique (up to normalization), then the whole family $\left(\varphi_{A}\right)$ converges to it as $A \rightarrow+\infty$. We gave in Remark 1.6 an example of a rotating field $v$ in the unit ball, for which the Rayleigh quotient has a unique minimizer (up to normalization) among all first integrals. This property could certainly be generalized to more general rotating type vector fields having at least one first integral in $\mathcal{I}_{0}$ whose level sets are connected hypersurfaces. We also showed in Remark 1.6 that the minimizers of this Rayleigh quotient among all first integrals may not be unique. But even in the example we gave in Remark 1.6, the whole family $\left(\varphi_{A}\right)$ still converges as $A \rightarrow+\infty$. One can wonder whether or not this convergence property always holds. Furthermore, this question of the convergence of the (normalized) first eigenvalues can also naturally be asked in the case where $v$ has no first integral.

Another natural question is about the other eigenvalues. For instance, for problem (0.1), we proved that the first eigenvalues are bounded if and only if the field $v$ has first integrals. Therefore, if there is no first integral, the other eigenvalues go to $+\infty$ as $A \rightarrow+\infty$. But if there are first integrals, can one say that the second eigenvalues are bounded as $A \rightarrow+\infty$ ? And so on for the other eigenvalues? We conjecture that the answer is yes.

Let us notice here that, under some additional regularity assumptions for $v$, the results of Theorem 0.3 could also be formally derived from the following variational formula by Holland [27] for the first eigenvalue $\lambda_{A}$ of problem (0.1), namely

$$
\lambda_{A}=\min _{\phi \in \Phi} \max _{V \in C^{1}(\Omega)} \frac{\int_{\Omega}|\nabla \phi|^{2}+A v \cdot(\nabla \phi+\phi \nabla V) \phi-|\nabla V|^{2} \phi^{2}}{\int_{\Omega} \phi^{2}},
$$

where $\Phi=\left\{\phi \in C^{2}(\Omega) \cap C(\bar{\Omega}), \phi>0\right.$ in $\Omega, \phi^{2}(x) / d(x) \rightarrow 0$ as $\left.x \rightarrow \partial \Omega, x \in \Omega\right\}$, and 
$d(x)$ is the distance of $x$ to $\partial \Omega$. At least under some smoothness assumptions, one has

$$
A \int_{\Omega} v \cdot(\nabla \phi+\phi \nabla V) \phi=A \int_{\Omega}(1-2 V)(v \cdot \nabla \phi) \phi .
$$

If $\phi \in \Phi$ is not a first integral of $v$, then there is an open set $\mathcal{U} \subset \Omega$ such that $v \cdot \nabla \phi$ does not vanish in $\mathcal{U}$. The choice $V=\left(1-\chi^{2} \phi v \cdot \nabla \phi\right) / 2$, where $\chi$ is a nonzero $C_{0}^{1}$ function whose support is included in $\mathcal{U}$, implies that, for such a $\phi$, the maximum with respect to $V$ in the right-hand side of (5.1) goes to $+\infty$ as $A \rightarrow+\infty$. This is a formal indication, but not a proof, suggesting that $\lambda_{A} \rightarrow+\infty$ as $A \rightarrow+\infty$ when $v$ has no first integral and that the $\lambda_{A}$ 's are expected to converge to the right-hand side of $(0.2)$ as $A \rightarrow+\infty$ if $v$ has first integrals.

The aforementioned questions or comments have their similar counterparts for the more general Dirichlet or Neumann/periodic problems (2.2) or (2.7).

Nevertheless, the simple question of finding a necessary and sufficient condition for the boundedness of the first eigenvalues remains open for similar elliptic problems with Robin type boundary conditions. The same question can also be asked for more general elliptic problems with non self-adjoint main part, even with Dirichlet boundary conditions. Namely, consider the following eigenvalue problem

$$
\left\{\begin{aligned}
-a_{i j} \partial_{i j} \varphi_{A}+A v \cdot \nabla \varphi_{A}+b_{i} \partial_{i} \varphi_{A}+c \varphi_{A} & =\lambda_{A} \varphi_{A} & & \text { in } \Omega \\
\varphi_{A} & =0 & & \text { on } \partial \Omega,
\end{aligned}\right.
$$

where $\Omega$ is a $C^{2}$ bounded domain of $\mathbb{R}^{N}, v$ is a bounded vector field such that $\operatorname{div} v=0$ in $\mathcal{D}^{\prime}(\Omega), b_{i} \in C(\bar{\Omega}), c=c(x) \in L^{\infty}(\Omega)$ and $a(x)=\left(a_{i j}(x)\right)_{1 \leq i, j \leq N}$ is a $C^{1}(\bar{\Omega})$ symmetric matrix field satisfying (2.1). Under these conditions, one can easily check that if there exists a sequence $\left(\lambda_{A_{n}}\right)_{n \in \mathbb{N}}$ which is bounded, then, after normalization in $L^{2}$ norm, a subsequence $\left(\varphi_{A_{n^{\prime}}}\right)_{n^{\prime}}$ converges strongly in $L^{2}$ and weakly in $H^{1}$ to a first integral of $v$ in $\mathcal{I}_{0}$. Conversely, is it true that if there is a first integral then the first eigenvalues are bounded ? Furthermore, even if a sequence $\left(\lambda_{A_{n}}\right)_{n \in \mathbb{N}}$ is bounded, does that imply that it converges, and that the whole family $\left(\lambda_{A}\right)$ converges as $A \rightarrow+\infty$ ? The answers are not clear.

To finish this section, let us mention that one can also ask about the generalizations of Theorems 0.3, 2.1 or 3.1 for elliptic or parabolic problems with large drift in unbounded domains with Dirichlet asymptotic conditions.

\section{References}

[1] D.G. Aronson, H.F. Weinberger, Multidimensional nonlinear diffusions arising in population genetics, Adv. Math. 30 (1978), pp 33-76.

[2] B. Audoly, H. Berestycki, Y. Pomeau, Réaction-diffusion en écoulement stationnaire rapide, C. R. Acad. Sci. Paris 328 II (2000), pp 255-262. 
[3] H. Berestycki, The influence of advection on the propagation of fronts in reaction-diffusion equations, In: Nonlinear PDE's in Condensed Matter and Reactive Flows, H. Berestycki and Y. Pomeau eds., Kluwer Academic Publ., 2002.

[4] H. Berestycki, F. Hamel, Front propagation in periodic excitable media, Comm. Pure Appl. Math. 55 (2002), pp 949-1032.

[5] H. Berestycki, F. Hamel, N. Nadirashvili, The speed of propagation for KPP type problems in periodic and more general domains, in preparation.

[6] H. Berestycki, B. Larrouturou, P.-L. Lions, Multidimensional traveling-wave solutions of a flame propagation model, Arch. Ration. Mech. Anal. 111 (1990), pp 33-49.

[7] H. Berestycki, L. Nirenberg, Travelling fronts in cylinders, Ann. Inst. H. Poincaré, Analyse Non Linéaire 9 (1992), pp 497-572.

[8] H. Berestycki, L. Nirenberg, S.R.S. Varadhan, The principal eigenvalue and maximum principle for second order elliptic operators in general domains, Comm. Pure Appl. Math. 47 (1994), pp 47-92.

[9] Y. Capdeboscq, Homogenization of a neutronic critical diffusion problem with drift, Proc. Royal Soc. Edinburgh 132 A (2002), pp 567-594.

[10] C. Conca, M. Vanninathan, Homogenization of periodic structures via Bloch decomposition, SIAM J. Appl. Math. 57 (1997), pp 1639-1659.

[11] P. Constantin, A. Kiselev, A. Oberman, L. Ryzhik, Bulk burning rate in passive-reactive diffusion, Arch. Ration. Mech. Anal. 154 (2000), pp 53-91.

[12] P. Constantin, A. Kiselev, L. Ryzhik, Quenching of flames by fluid advection, Comm. Pure Appl. Math. 54 (2001), pp 1320-1342.

[13] A. Devinatz, R. Ellis, A. Friedman, The asymptotic behavior of the first real eigenvalue of the second-order elliptic operator with a small parameter in the higher derivatives, II, Indiana Univ. Math. J. (1973/74), pp 991-1011.

[14] A. Fannjiang, G. Papanicolaou, Convection enhanced diffusion for periodic flows, SIAM J. Appl. Math. 54 (1994), pp 333-408.

[15] P.C. Fife, Mathematical aspects of reacting and diffusing systems, Lecture Notes in Biomathematics 28, Springer Verlag, 1979.

[16] R.A. Fisher, The advance of advantageous genes, Ann. Eugenics 7 (1937), pp 335-369.

[17] W.H. Fleming, S.-J. Sheu, Asymptotics for the principal eigenvalue and eigenfunction of a nearly first-order operator with large potential, Ann. Probab. 25 (1997), pp 1953-1994.

[18] M. Freidlin, Functional integration and partial differential equations, Ann. of Math. Studies, Princeton University Press, 1985.

[19] M. Freidlin, Wave front propagation for KPP type equations, Surveys in Appl. Math. 2, Plenum, New York, 1995, pp 1-62. 
[20] A. Friedman, The asymptotic behavior of the first real eigenvalue of a second order elliptic operator with a small parameter in the highest derivatives, Indiana Univ. Math. J. 22 (1973), pp 1005-1015.

[21] J. Gärtner, M. Freidlin, On the propagation of concentration waves in periodic and random media, Sov. Math. Dokl. 20 (1979), pp 1282-1286.

[22] F. Hamel, Formules min-max pour les vitesses d'ondes progressives multidimensionnelles, Ann. Fac. Sci. Toulouse 8 (1999), pp 259-280.

[23] S. Heinze, Homogenization of flame fronts, Preprint IWR, Heidelberg, 1993.

[24] S. Heinze, Wave solutions for reaction-diffusion systems in perforated domains, Z. Anal. Anwendungen 20 (2001), pp 661-670.

[25] S. Heinze, The speed of travelling waves for convective reaction-diffusion equations, Preprint MPI, Leipzig, 2001.

[26] S. Heinze, G. Papanicolaou, A. Stevens, Variational principles for propagation speeds in inhomogeneous media, SIAM J. Appl. Math. 62 (2001), pp 129-148.

[27] C.J. Holland, A minimum principle for the principal eigenvalue for second-order linear elliptic equations with natural boundary conditions, Comm. Pure Appl. Math. 31 (1978), pp 509-519.

[28] W. Hudson, B. Zinner, Existence of travelling waves for reaction-diffusion equations of Fisher type in periodic media, In: Boundary Value Problems for Functional-Differential Equations, J. Henderson (ed.), World Scientific, 1995, pp 187-199.

[29] Ya.I. Kanel', Certain problems of burning-theory equations, Sov. Math. Dokl. 2 (1961), pp 48-51.

[30] A. Kiselev, L. Ryzhik, Enhancement of the traveling front speeds in reaction-diffusion equations with advection, Ann. Inst. H. Poincaré, Analyse Non Linéaire 18 (2001), pp 309-358.

[31] A.N. Kolmogorov, I.G. Petrovsky, N.S. Piskunov, Étude de l'équation de la diffusion avec croissance de la quantité de matière et son application à un problème biologique, Bulletin Université d'État à Moscou (Bjul. Moskowskogo Gos. Univ.), Série internationale A 1 (1937), pp 1-26.

[32] A.J. Majda, P.E. Souganidis, Large scale front dynamics for turbulent reaction-diffusion equations with separated velocity scales, Nonlinearity 7 (1994), pp 1-30.

[33] N. Shigesada, K. Kawasaki, E. Teramoto, Spatial segregation of interacting species, J. Theoret. Biol. 79 (1979), pp 83-99.

[34] A.I. Volpert, V.A. Volpert, V.A. Volpert, Traveling wave solutions of parabolic systems, Translations of Math. Monographs 140, Amer. Math. Soc., 1994.

[35] X. Xin, Existence of planar flame fronts in convective-diffusive periodic media, Arch. Ration. Mech. Anal. 121 (1992), pp 205-233.

[36] J.X. Xin, Analysis and modeling of front propagation in heterogeneous media, SIAM Review 42 (2000), pp 161-230. 
[37] A.D. Wentzell, On the asymptotic behavior of the first eigenvalue of a second-order differential operator with small parameter in higher derivatives, Theory Probab. Appl. 20 (1975), pp 599602.

[38] A.D. Wentzell, M. Freidlin, Random perturbations of dynamical systems, Grund. Math. Wissenschaften, second ed., Springer : New York, 1998. 